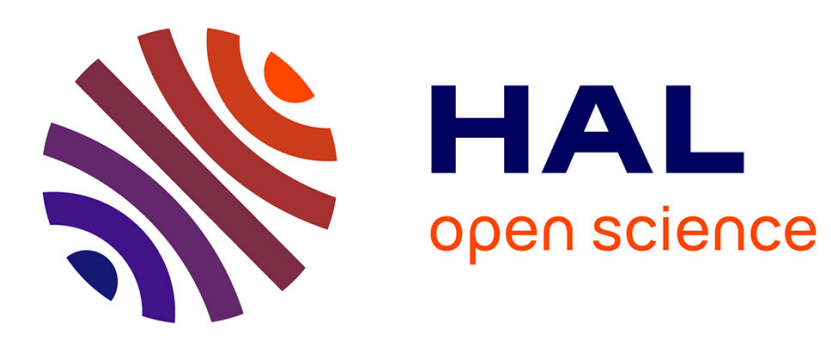

\title{
STAP à Rand Réduit, Robuste et Persymétrique
}

Guillaume Ginolhac, Philippe Forster, Jean-Philippe Ovarlez, Frédéric Pascal

\section{To cite this version:}

Guillaume Ginolhac, Philippe Forster, Jean-Philippe Ovarlez, Frédéric Pascal. STAP à Rand Réduit, Robuste et Persymétrique. Traitement du Signal, 2011, 28 (1-2), pp.143-170. 10.3166/ts.28.143-170 . hal-00659841

\section{HAL Id: hal-00659841 \\ https://hal-centralesupelec.archives-ouvertes.fr/hal-00659841}

Submitted on 4 Mar 2020

HAL is a multi-disciplinary open access archive for the deposit and dissemination of scientific research documents, whether they are published or not. The documents may come from teaching and research institutions in France or abroad, or from public or private research centers.
L'archive ouverte pluridisciplinaire HAL, est destinée au dépôt et à la diffusion de documents scientifiques de niveau recherche, publiés ou non, émanant des établissements d'enseignement et de recherche français ou étrangers, des laboratoires publics ou privés. 


\section{STAP à Rang Réduit, Robuste et Persymétrique}

\section{Guillaume Ginolhac ${ }^{*}$ - Philippe Forster ${ }^{*}$ - Jean-Philippe Ovarlez $^{* * * * * *}$ - Frédéric Pascal ${ }^{*}$}

* SATIE

ENS CACHAN, CNRS, UniverSud

61 av du President Wilson

94235 CACHAN Cedex

\{guillaume.ginolhac,philippe.forster\}@satie.ens-cachan.fr

** ONERA (DEMR/TSI)

Département Electromagnétisme et Radar - Unité Traitement du Signal

Chemin de la Hunière

91761 Palaiseau Cedex

ovarlez@onera.fr

*** SONDRA

Supélec

Plateau du Moulon, 3 rue Joliot-Curie

91192 Gif-sur-Yvette Cedex

frederic.pascal@supelec.fr

RÉSUMÉ. Dans ce papier, nous proposons trois filtres STAP rang faible et les détecteurs adaptatifs correspondants dans le cadre d'un bruit composé d'un clutter rang faible de distribution Spherically Invariant Random Vector (SIRV) plus d'un bruit blanc Gaussien. Les projecteurs orthogonaux au sous espace fouillis sont construits à partir de la Sample Covariance Matrix (SCM), de la SCM persymétrique (utilisant les propriétés de symétrie spatiales et temporelles du RADAR) et de la Normalized Sample Covariance Matrix (NSCM). Nous présentons les performances de ces trois filtres en terme de pertes en Rapport Signal à Bruit (RSB) à l'aide d'une analyse de perturbation. Nous étudions aussi la robustesse de ces filtres STAP à la contamination des données secondaires par des composants de la cible. Ces différentes propriétés sont finalement vérifiées sur les données CELAR.

ABSTRACT. In this paper, we propose three Low-Rank (LR) STAP Filters and the corresponding adaptive detectors when the disturbance is modelled as the sum of a Spherically Invariant Ran-

$1^{\text {re }}$ soumission à Traitement du signal, le 15 juillet 2011 
dom Vector (SIRV) and a white noise Gaussian. The orthogonal projectors onto the subspace clutter are built from the Sample Covariance Matrix (SCM), the persymmetric SCM (by using the temporal and/or spatial symmetric properties of the RADAR system) and the Normalized Sample Covariance Matrix (NSCM). We present the theoretical performances of these three filters based on the statistical law of Signal to Noise Ratio (SNR) Loss computed from an analysis of perturbation. We also study the robustness of these filters to the secondary data contamination by target components. These different properties are finally checked on the data DGA/MI MOTS-CLÉS : filtrage, rang faible, SIRV, Normalized Sample Covariance Matrix, persymétrie KEYWORDS: filtering, low-rank, SIRV, Normalized Sample Covariance Matrix, persymmetry 


\section{Extended Abstract}

Space Time Adaptive Processing (STAP) is a technique used in airborne phased array radar to detect moving target embedded in an interference background such as jamming or strong clutter (Ward, 1994). While conventional radars are capable of detecting targets both in the time domain related to target range and in the frequency domain related to target velocity, STAP uses an additional domain (space) related to the target angular localization. The consequence is a two-dimensional adaptive filtering technique which uses jointly temporal and spatial dimensions to suppress interference and to improve target detection. In most works on radar, the clutter is assumed to be a lonely Gaussian process. But, the increase of the radar resolution leads to a higher scene heterogeneity where the clutter can no longer be modeled by a Gaussian process (Conte et al., 1997; Billingsley et al., 1999; Greco et al., 2006). To take this heterogeneity into account, one can use the Spherically Invariant Random Vector (SIRV) product model, first introduced by Yao (Yao, 1973) for the information theory. This is a compound-Gaussian model, well-known for its good statistical properties as well as it has been validated on several real data sets (Watts, 1985; Nohara et al., 1991; Billingsley, 1993; Conte et al., 2004). Furthermore, this model encompasses an infinity of distributions. Among them, there is of course the Gaussian distribution and one can also cite the K-distribution widely used in the radar processing (Gini, 1997), the Weibull distribution or the Rician one. In classical STAP context (as side-looking), the ground clutter can be considered to have a Low Rank (LR) structure from the Brennan's Rule (Brennan et al., 1992). Therefore, the disturbance in this paper is modeled as the sum of a LR-SIRV clutter and a zero-mean white Gaussian noise. It is a general model as considered in (Rangaswamy et al., 2004).

In practice, the disturbance covariance matrix is generally unknown and an estimate is required to perform the STAP processing. In the context of LR clutter, only the estimation of the clutter subspace projector is needed for the processing (Kirsteins et al., 1994; Haimovich, 1996). This estimation procedure requires the so-called secondary data. These data are assumed to be independent and share the same distribution as the observation under test. In a STAP framework, the dimension of the covariance matrix is important (number of sensors times number of pulses). Commonly, the number of secondary data has to be upper than two times this dimension to ensure the classical 3dB loss for the performance results (Reed et al., 1974). One of the advantage of the LR techniques is that this rule can be strongly relaxed to preserve such a performance (Kirsteins et al., 1991; Kirsteins et al., 1994; Haimovich, 1997; Peckham et al., 2000).

To improve the estimation quality of the projector based on the SCM, it is possible to use some structural properties of the covariance matrix. Actually, STAP radar have often symmetric properties in the spatial and / or temporal dimension (Cai et al., 1992; Conte et al., 2003). In this case, the clutter plus white noise covariance matrix and the spatio-temporal vector present the persymetric property (Ovarlez et al., 2011). The maximum likelihood estimation of the covariance matrix leads to a real matrix (Pailloux et al., 2008) which allows to double the number of sensors. It 
seems to be interesting to build the projector from this new estimator of the covariance matrix because it will allow to reduce the number of secondary data needed to a correct estimation. Another problem in STAP comes from these secondary data which are often contaminated by the secondary lobes of the target under study or other targets with same angular and velocity properties. It is also possible to have outliers in the STAP data cube like for example in highway STAP data with cars traffic-jam or convoys tracking. In this case, LR techniques exhibit another advantage on classical methods: for a quite low Signal-to-Clutter Ratio (SCR), LR techniques are robust to secondary data contamination, see e.g., (Kirsteins et al., 1994; Ginolhac et al., 2002). This robustness is directly relied on the choice of the covariance matrix estimate, from which the projector estimate will be derived. It seems obvious that the well-known Sample Covariance Matrix (SCM) is not adapted to strong contamination problems since this estimate depends on the power of each data. Thus, it is interesting to consider other covariance matrix estimates, independent to the data power, i.e. self-normalized estimates. This is the case of the Normalized Sample Covariance Matrix (NSCM) (Gini et al., 1995) and the Fixed Point Estimate (FPE) (Gini et al., 2002; Conte et al., 2002). Even if the FPE exhibits better statistical properties than the NSCM (Pascal et al., 2008b) (NSCM is a biased estimate of the covariance matrix) and provides an adaptive detector with the SIRV-CFAR property (texture and covariance matrix CFAR) (Pascal et al., 2006), the FPE is not adapted to LR context (it requires an inversion of matrix, which is impossible in the case of a small number of secondary data). We then proposed in (Ginolhac et al., 2009) to build the clutter subspace projector on the NSCM. The corresponding LR STAP filter seems to exhibit interesting results: it is more robust to secondary data contamination by target components than the LR STAP filter obtained from the SCM. Moreover, we showed in (Ginolhac et al., 2011) that the projector onto the clutter subspace built from the NSCM is a consistent estimate of the true one when the disturbance is modelled as the sum of a LR-SIRV clutter and a white Gaussian noise.

When disturbance is modelled by the sum of LR-Gaussian clutter plus a white Gaussian noise, performances of LR STAP filter based on SCM are computed in (Kirsteins et al., 1991; Kirsteins et al., 1994; Haimovich, 1997; Peckham et $a l ., 2000)$ and those of LR STAP filter based on NSCM are given in (Ginolhac et al., 2010). We propose in this paper to compute the theoretical performances of the LR-STAP filters built from the SCM and the NSCM in a LR-SIRV clutter plus white Gaussian noise. This study is based on a study of the Signal to Noise Ratio (SNR) Loss and a perturbation analysis (Krim et al., 1992). We propose to validate the theoretical results by numerical simulations which also allow to compare the SNR Loss obtained from the LR-STAP filters built from SCM and NSCM. In this paper, we also illustrate the property of the better robustness of the LR-STAP filter built from the NSCM than the one built from the SCM by a discussion and simulation with real clutter and synthetic targets. Even if several works show the interest to select the secondary data (Rabideau et al., 1999; Gerlach et al., 2004; Guerci et al., 2000) to avoid outliers, the LR-STAP filter built from the NSCM is simple method to obtain a good 
robustness to target contamination by target components. Both methods could be used at the same time.

In this paper, we propose to develop the Low-Rank STAP filters with the projector onto the clutter subspace is built from either the SCM, or the persymetric SCM or the NSCM. By using (Ginolhac, 2011; Ginolhac et al., 2012; Pailloux, 2010), we present the performances of these Low-Rank STAP filters in the context of a SIRV clutter plus a white Gaussian noise. These computations are based on a perturbation analysis (Krim et al., 1992) which consists to obtain a perturbator to model the estimation error of covariance matrix estimators. We also study the interest of the NSCM for the robustness to the contamination of secondary data by targets. For this study, we propose several adaptive detectors based on Low-Rank STAP filters calculated with the three covariance matrix estimators. Results on DGA/MI (Bidon et al., 2011) data allow to study the impact of the low-rank structure and the persymetry on the number of secondary data used for the projector estimation. We also illustrate the robustness of these filters to the to the contamination of secondary data by targets. 
$61^{\text {re }}$ soumission à Traitement du signal 


\section{Introduction}

Le STAP (Space Time Adaptive Processing) est une technique récente (Ward, 1994; Klemm, 2002) utilisant une antenne aéroportée dans le but de détecter une cible en mouvement noyée dans un bruit complexe composé de plusieurs éléments tels que le jamming (causé par un brouilleur à une direction donnée), le fouillis (causé par le sol) ou le bruit blanc (causé par l'électronique). Alors que les radar conventionnels sont capables de détecter les cibles dans le domaine temporel relié à la distance de la cible et dans le domaine fréquentiel relié à la vitesse de la cible, le STAP utilise une dimension supplémentaire, le domaine spatial, relié à la localisation angulaire de la cible. La conséquence de ce nouveau type de radar est un filtre bi-dimensionnel adaptatif qui utilise en même temps les dimensions temporelle et spatiale dans le but de supprimer les interférences et d'améliorer la détection de la cible. Dans le cadre de ces travaux, nous ne considérons pas le problème du jamming mais seulement du fouillis de sol et du bruit blanc.

Dans la majorité des travaux sur le radar, le bruit total est modélisé comme un simple processus Gaussien sans plus d'informations sur sa structure. Mais d'après la formule de Brennan (Brennan et al., 1992), le fouillis de sol en STAP (dans les configurations classiques) a un rang faible, le bruit total a alors une structure particulière et peut être modélisé comme la somme d'un fouillis rang faible provenant de la rétrodiffusion du signal émis sur le sol et d'un bruit blanc modélisant le bruit de l'électronique. De plus, le modèle Gaussien pour le fouillis n'est pas toujours adapté aux données rencontrées dans la réalité comme dans le cas du STAP Haute Résolution (STAP-HR). En effet, la puissance du fouillis peut alors varier fortement d'une case distance à l'autre (caractère non stationnaire). Dans ce cas, il est usuel dans la littérature de modéliser le fouillis comme un Spherically Invariant Random Vector (SIRV) (Yao, 1973; Rangaswamy et al., 1993) pour ses bonnes propriétés statistiques et pour sa bonne correspondance avec les données réelles (Watts, 1985; Nohara et al., 1991; Billingsley, 1993). Ce modèle inclut les distributions classiques comme par exemple la distribution gaussienne, la K-distribution (Gini, 1997) ou la distribution de Weibull. Dans cette étude, nous considérons donc que l'interférence est la somme d'un fouillis rang faible SIRV et d'un bruit blanc Gaussien centré. Ce modèle est le même que celui considéré dans (Rangaswamy et al., 2004).

Le traitement STAP nécessite l'estimation de la matrice de covariance de l'interférence qui est généralement inconnue. Dans le cadre d'un fouillis rang faible, l'estimation du projecteur sur sous espace fouillis est suffisant. Ces estimations sont faites à partir de données, appelées données secondaires, supposant partager la même distribution statistique que la donnée sous test. Il est connu que les algorithmes adaptatifs basés sur une modélisation rang faible nécessitent beaucoup moins de données secondaires que les algorithmes classiques (Kirsteins et al., 1991; Kirsteins et al., 1994; Haimovich, 1997). L'estimation du projecteur se fait classiquement à l'aide de la Sample Covariance Matrix (SCM). 
Pour améliorer la qualité de l'estimation du projecteur basé sur la SCM, il est possible d'utiliser certaines propriétés structurelles de la matrice de covariance. En effet, les radars STAP présentent souvent des propriétés de symétrie dans les domaines spatiaux et / ou temporels (Cai et al., 1992; Conte et al., 2003). Dans ce cas, la matrice de covariance du clutter plus du bruit blanc et le vecteur spatio-temporel présentent la propriété de persymétrie (propriété de symétrie de la matrice de covariance par rapport à l'antidiagonale) (Ovarlez et al., 2011). Alors, l'estimation par le maximum de vraisemblance de la matrice de covariance conduit à une matrice réelle (Pailloux et $a l ., 2008)$ ce qui permet de doubler artificiellement le nombre de capteurs. Il semble donc intéressant de construire le projecteur à partir de ce nouvel estimateur de la matrice de covariance, car il devrait permettre de pouvoir réduire le nombre de données secondaires nécessaires à une estimation correcte. Malheureusement, il arrive aussi parfois que les données secondaires soient contaminées par des cibles secondaires soit parce que la cible sous étude bave sur plusieurs cases, soit car différentes cibles avec les mêmes propriétés de direction et de vitesse sont présentes (données autoroutes). Dans ce cadre, un autre avantage des méthodes rang faible par rapport aux méthodes classiques est leur robustesse à cette contamination des données secondaires par la cible (Kirsteins et al., 1994; Ginolhac et al., 2002) dans l'hypothèse d'un Rapport Signal à fouillis (RSC) faible. Mais il semble évident que dans le cadre d'une contamination par une cible forte, celle-ci prenne trop d'importance dans la SCM (et donc l'hypothèse de faible RSC n'est plus vérifiée). Il est donc intéressant d'utiliser d'autres estimateurs de la matrice de covariance faisant intervenir une normalisation comme la Normalized Sample Covariance Matrix (NSCM) (Gini et al., 1995) et la Matrice du Point Fixe (MPF) (Gini et al., 2002; Conte et al., 2002). La MPF est connue comme ayant de meilleures propriétés statistiques que la NSCM car elle n'est pas biaisée et qu'elle est efficace (Pascal et al., 2008b) et permet donc d'avoir un détecteur, dans le cas de la détection d'une cible noyée dans un fouillis SIRV, R-CFAR (Constant False Alarm on Covariance Matrix) (Pascal et al., 2006). Mais la procédure itérative, qui est déjà d'une complexité de calcul importante, pour obtenir la MPF (Pascal et al., 2008a) est malheureusement impossible dans le cas de peu données secondaires (ce qui est intéressant dans le cadre d'un fouillis rang faible) à cause de considérations algébriques.

Nous proposons donc dans ce papier de développer des filtres STAP rang faible pour lequels le projecteur sur le sous espace fouillis est construit à partir soit de la SCM, soit de la SCM persymétrique ou soit de la NSCM. En utilisant (Ginolhac, 2011; Ginolhac et al., 2012; Pailloux, 2010), nous présentons les performances de ces filtres STAP rang faible dans le cadre d'un fouillis SIRV plus un bruit blanc Gaussien centré. Ces calculs sont basés sur une analyse de perturbation (Krim et al., 1992) qui consiste à calculer un perturbateur pour modéliser l'erreur d'estimation des estimateurs de la matrice de covariance (et donc des projecteurs correspondants). Nous étudions aussi l'apport de la NSCM pour la robustesse à la contamination des données secondaires par des données cibles. Pour cela, nous proposons plusieurs détecteurs adaptatifs basés sur les filtres STAP rang faible calculés à partir des trois estimateurs de la matrice de covariance. Les résultats sur les données DGA/MI (Bidon et al., 2011) 
vont nous permettre d'étudier l'impact de la structure rang faible et de la persymétrie sur le nombre de données secondaires utilisées pour l'estimation du projecteur. Nous illustrerons aussi la robustesse de ces filtres à la contamination des données secondaires par des composants de la cible.

La section 2 présente le modèle du signal reçu, le problème de détection, la structure de la matrice de covariance ainsi que les filtres et détecteurs optimaux correspondants. Cette section se divise en deux sous sections en fonction de la prise en compte de la persymétrie. La section 3 présente nos trois méthodes pour estimer le projecteur orthogonal au sous espace fouillis. La section 4 est consacrée aux versions adaptatives des détecteurs alors que la section 5 montre les résultats de ces détecteurs sur les données DGA/MI.

Les conventions suivantes sont adoptées : l'italique indique une quantité scalaire, une minuscule en gras indique un vecteur et une majuscule en gras indique une matrice. ${ }^{T}$ correspond à l'opérateur de transposition et ${ }^{H}$ à la transposition conjuguée. $E$ [ ] est l'opérateur d'espérance mathématique. $\operatorname{tr}(\boldsymbol{A})$ est l'opérateur calculant la trace de la matrice $\boldsymbol{A} . \boldsymbol{I}_{m}$ est la matrice identité de taille $m \times m . \mathcal{C N}(\boldsymbol{m}, \boldsymbol{R})$ représente un vecteur aléatoire de loi gaussienne complexe de moyenne $\boldsymbol{m}$ et de matrice de covariance $\boldsymbol{R}$. \|\|$_{F}$ correspond à la norme de Froebenius.

\section{Modèle du signal et filtres / détecteurs STAP optimaux dans un contexte de fouillis rang faible}

\subsection{Modèle du signal STAP dans un contexte de fouillis rang faible sans tenir compte de la propriété de la persymétrie}

Dans un cadre de détection en STAP, le problème est de détecter un signal complexe $\boldsymbol{d}$ corrompu par un bruit additif $\boldsymbol{c}+\boldsymbol{n}$ dans un vecteur d'observation $\boldsymbol{x} \in \mathbb{C}^{m}$ ( $m=N M$ où $N$ est le nombre de voies spatiales de l'antenne et $M$ le nombre d'impulsions sur une rafale). Ceci peut être résumé selon le test d'hypothèse binaire suivant :

$$
\left\{\begin{array}{lll}
H_{0}: \boldsymbol{x}=\boldsymbol{c}+\boldsymbol{n} & \boldsymbol{x}_{k}=\boldsymbol{c}_{k}+\boldsymbol{n}_{k} & k=1, \ldots, K \\
H_{1}: \boldsymbol{x}=\boldsymbol{d}+\boldsymbol{c}+\boldsymbol{n} & \boldsymbol{x}_{k}=\boldsymbol{c}_{k}+\boldsymbol{n}_{k} & k=1, \ldots, K
\end{array},\right.
$$

où les $\boldsymbol{x}_{k} \in \mathbb{C}^{m}$ sont les $K$ données secondaires qui sont supposées sans composants du signal. Le signal complexe $\boldsymbol{d}$ peut être décomposé comme $\boldsymbol{d}=\alpha \boldsymbol{a}\left(\theta, f_{d}\right)$ où $\boldsymbol{a}$ est le vecteur spatio-temporel (parfaitement connu), $\theta$ est l'angle d'arrivée, $f_{d}$ est la fréquence Doppler et $\alpha$ l'amplitude complexe. Les paramètres $\theta, f_{d}$ et $\alpha$ sont inconnus.

Le bruit blanc $\boldsymbol{n}$ (ou $\boldsymbol{n}_{k}$ ) est modélisé par un vecteur aléatoire complexe gaussien $\mathcal{C N}\left(\mathbf{0}, \lambda \boldsymbol{I}_{m}\right)$ centré blanc de matrice de covariance $\lambda \boldsymbol{I}_{m}$. Nous supposons dans ce papier que la puissance du fouillis dans chaque cellule $k$ et dans la cellule test est différent. Dans une telle situation, il est classique d'utiliser comme modèle les processus 
SIRV (Yao, 1973; Rangaswamy et al., 1993; Pascal et al., 2008a; Pascal et al., 2006). Un SIRV est un vecteur aléatoire gaussien non-homogène car sa puissance est aléatoire : cela traduit une variation spatiale du retour du radar. Le SIRV (Yao, 1973) $c$ (resp. $\boldsymbol{c}_{k}$ ) est alors le produit d'une variable aléatoire positive $\tau$ (resp. $\tau_{k}$ ), notée texture, et d'un vecteur complexe gaussien de dimension $m \boldsymbol{g}$ (resp. $\boldsymbol{g}_{k}$ ), noté speckle, de loi $\mathcal{C N}(\mathbf{0}, \boldsymbol{C})$ avec $\boldsymbol{C}=E\left[\boldsymbol{g g}^{H}\right]=E\left[\boldsymbol{g}_{k} \boldsymbol{g}_{k}^{H}\right]$. On peut alors écrire $\boldsymbol{c}$ (resp. $\boldsymbol{c}_{k}$ ) sous la forme suivante :

$$
\begin{aligned}
\boldsymbol{c} & =\sqrt{\tau} \boldsymbol{g} \\
\boldsymbol{c}_{k} & =\sqrt{\tau}_{k} \boldsymbol{g}_{k} \quad k=1, \ldots, K
\end{aligned}
$$

Notons que la matrice de covariance est normalisée selon $\operatorname{tr}(\boldsymbol{C})=m$ (voir (Gini et al., 2002)) pour des considérations d'identifiabilité. La matrice de covariance de $\boldsymbol{c}+\boldsymbol{n}$ (ou $\boldsymbol{c}_{k}+\boldsymbol{n}_{k}$ ) s'écrit alors :

$$
\boldsymbol{\Sigma}=E(\tau) \boldsymbol{C}+\lambda \boldsymbol{I}_{m}
$$

Dans un contexte de fouillis Gaussien, nous avons $\tau=1$.

Dans le contexte du STAP classique (par exemple en mode side-looking), nous pouvons évaluer le rang du fouillis à l'aide de la formule de Brennan (Brennan et al., 1992) qui nous assure une structure rang réduit pour le fouillis :

$$
\operatorname{rang}(\boldsymbol{C})=r \ll m
$$

La matrice de covariance du speckle $C$ peut alors se décomposer selon :

$$
\boldsymbol{C}=\sum_{i=1}^{r} \lambda_{i} \boldsymbol{u}_{i} \boldsymbol{u}_{i}^{H}
$$

où $\lambda_{1}>\lambda_{2}>\ldots>\lambda_{r}>\lambda_{r+1}=\ldots=\lambda_{m}=0$ sont les valeurs propres de $\boldsymbol{C}$ et $\left\{\boldsymbol{u}_{1}, \ldots, \boldsymbol{u}_{r}, \boldsymbol{u}_{r+1}, \ldots, \boldsymbol{u}_{m}\right\}$ sont les vecteurs propres associés. La décomposition de $\boldsymbol{\Sigma}$ s'écrit alors :

$$
\boldsymbol{\Sigma}=\sum_{i=1}^{r}\left(E[\tau] \lambda_{i}+\lambda\right) \boldsymbol{u}_{i} \boldsymbol{u}_{i}^{H}+\lambda \sum_{i=r+1}^{m} \boldsymbol{u}_{i} \boldsymbol{u}_{i}^{H}
$$

Définissons les deux matrices unitaires suivantes :

$$
\begin{aligned}
\boldsymbol{U}_{r} & =\left[\boldsymbol{u}_{1} \ldots \boldsymbol{u}_{r}\right] \\
\boldsymbol{U}_{0} & =\left[\boldsymbol{u}_{r+1} \ldots \boldsymbol{u}_{m}\right]
\end{aligned}
$$

Nous définissons le projecteur sur le sous espace fouillis $\Pi_{c}$ et le projecteur orthogonal au sous espace fouillis $\Pi_{c}^{\perp}$ (Kirsteins et al., 1994; Haimovich, 1997) :

$$
\begin{aligned}
& \boldsymbol{\Pi}_{c}=\boldsymbol{U}_{r} \boldsymbol{U}_{r}^{H}=\sum_{i=1}^{r} \boldsymbol{u}_{i} \boldsymbol{u}_{i}^{H} \\
& \boldsymbol{\Pi}_{c}^{\perp}=\boldsymbol{U}_{0} \boldsymbol{U}_{0}^{H}=\boldsymbol{I}_{m}-\boldsymbol{\Pi}_{c}=\sum_{i=r+1}^{m} \boldsymbol{u}_{i} \boldsymbol{u}_{i}^{H}
\end{aligned}
$$




\subsection{Modèle du signal STAP dans un contexte de fouillis rang faible en tenant compte de la propriété de la persymétrie}

Dans beaucoup de systèmes radar, il est courant de rencontrer des antennes symétriques et/ou des trains d'impulsions symétriques (Cai et al., 1992; Conte et al., 2003). Dans ce cadre, la vraie matrice de covariance $\Sigma$ de l'Eq. [3] possède la propriété de persymétrie suivante :

$$
\boldsymbol{\Sigma}=\boldsymbol{J}_{m} \boldsymbol{\Sigma}^{*} \boldsymbol{J}_{m},
$$

où $\boldsymbol{J}_{m}$ est la matrice antidiagonale de taille $m \times m$ (contient des 1 sur l'antidiagonale et des 0 ailleurs). Le vecteur spatio-temporel est aussi persymétrique :

$$
\boldsymbol{d}=\boldsymbol{J}_{m} \boldsymbol{d}^{*}
$$

Pour utiliser cette propriété, nous devons transformer les données complexes du problème de détection [1] en données réelles. Pour faire cette transformation, définissons la matrice unitaire $\boldsymbol{T}$ :

$$
\boldsymbol{T}=\left\{\begin{array}{l}
\frac{1}{\sqrt{2}}\left(\begin{array}{cc}
\boldsymbol{I}_{m / 2} & \boldsymbol{J}_{m / 2} \\
i \boldsymbol{I}_{m / 2} & i \boldsymbol{J}_{m / 2}
\end{array}\right) m \text { pair } \\
\frac{1}{\sqrt{2}}\left(\begin{array}{ccc}
\boldsymbol{I}_{(m-1) / 2} & 0 & \boldsymbol{J}_{(m-1) / 2} \\
0 & \sqrt{2} & 0 \\
i \boldsymbol{I}_{(m-1) / 2} & 0 & i \boldsymbol{J}_{(m-1) / 2}
\end{array}\right) m \text { impair }
\end{array}\right.
$$

Maintenant, définissons les données transformées par $\boldsymbol{T}$ :

$$
\begin{aligned}
\boldsymbol{x}^{\prime} & =\boldsymbol{T} \boldsymbol{x} \\
\boldsymbol{x}_{k}^{\prime} & =\boldsymbol{T} \boldsymbol{x}_{k} \\
\boldsymbol{d}^{\prime} & =\boldsymbol{T} \boldsymbol{d} \\
\boldsymbol{a}^{\prime} & =\boldsymbol{T} \boldsymbol{a} \\
\boldsymbol{c}^{\prime} & =\boldsymbol{T} \boldsymbol{c} \\
\boldsymbol{c}_{k}^{\prime} & =\boldsymbol{T} \boldsymbol{c}_{k} \\
\boldsymbol{n}^{\prime} & =\boldsymbol{T} \boldsymbol{n} \\
\boldsymbol{n}_{k}^{\prime} & =\boldsymbol{T} \boldsymbol{n}_{k}
\end{aligned}
$$

Le problème de détection [1] devient alors après transformation par $\boldsymbol{T}$ :

$$
\left\{\begin{array}{lll}
H_{0}: \boldsymbol{x}^{\prime}=\boldsymbol{c}^{\prime}+\boldsymbol{n}^{\prime} & \boldsymbol{x}_{k}^{\prime}=\boldsymbol{c}_{k}^{\prime}+\boldsymbol{n}_{k}^{\prime} & k=1, \ldots, K \\
H_{1}: \boldsymbol{x}^{\prime}=\boldsymbol{d}^{\prime}+\boldsymbol{c}^{\prime}+\boldsymbol{n}^{\prime} & \boldsymbol{x}_{k}^{\prime}=\boldsymbol{c}_{k}^{\prime}+\boldsymbol{n}_{k}^{\prime} & k=1, \ldots, K
\end{array},\right.
$$

où le signal réel $\boldsymbol{d}^{\prime}$ peut être décomposé comme $\boldsymbol{d}^{\prime}=\alpha \boldsymbol{a}^{\prime}(\theta, f)$.

La matrice de covariance du fouillis plus du bruit blanc est alors de la forme :

$$
\Sigma^{\prime}=T \Sigma T^{H}
$$

La décomposition de $\boldsymbol{\Sigma}^{\prime}$ s'écrit alors :

$$
\boldsymbol{\Sigma}^{\prime}=\sum_{i=1}^{r}\left(E[\tau] \lambda_{i}+\lambda\right) \boldsymbol{u}_{i}^{\prime} \boldsymbol{u}_{i}^{\prime H}+\lambda \sum_{i=r+1}^{m} \boldsymbol{u}_{i}^{\prime} \boldsymbol{u}_{i}^{\prime H}
$$


où $\left\{\boldsymbol{u}_{1}^{\prime}, \ldots, \boldsymbol{u}_{r}^{\prime}, \boldsymbol{u}_{r+1}^{\prime}, \ldots, \boldsymbol{u}_{m}^{\prime}\right\}$ sont les vecteurs propres de $\boldsymbol{\Sigma}^{\prime}$. On remarque le rang de la matrice de covariance reste inchangé par l'opérateur $\boldsymbol{T}$.

Définissons les deux matrices unitaires suivantes :

$$
\begin{aligned}
\boldsymbol{U}_{r}^{\prime} & =\left[\boldsymbol{u}_{1}^{\prime} \ldots \boldsymbol{u}_{r}^{\prime}\right] \\
\boldsymbol{U}_{0}^{\prime} & =\left[\boldsymbol{u}_{r+1}^{\prime} \ldots \boldsymbol{u}_{m}^{\prime}\right]
\end{aligned}
$$

Nous définissons le projecteur sur le sous espace fouillis $\Pi_{c}^{\prime}$ et le projecteur orthogonal au sous espace orthogonal $\Pi_{c}^{\prime \perp}$ (Kirsteins et al., 1994; Haimovich, 1997) :

$$
\begin{aligned}
& \boldsymbol{\Pi}_{c}^{\prime}=\boldsymbol{U}_{r}^{\prime} \boldsymbol{U}_{r}^{\prime H}=\sum_{i=1}^{r} \boldsymbol{u}_{i}^{\prime} \boldsymbol{u}_{i}^{\prime H} \\
& \boldsymbol{\Pi}_{c}^{\perp \perp}=\boldsymbol{U}_{0}^{\prime} \boldsymbol{U}_{0}^{\prime H}=\boldsymbol{I}_{m}-\boldsymbol{\Pi}_{c}^{\prime}=\sum_{i=r+1}^{m} \boldsymbol{u}_{i}^{\prime} \boldsymbol{u}_{i}^{\prime H}
\end{aligned}
$$

\subsection{Les Filtres et les tests de détection optimaux dans un contexte de fouillis rang faible sans tenir compte de la propriété de la persymétrie}

Nous présentons tout d'abord le filtre STAP optimal :

$$
\boldsymbol{w}_{\text {opt }}=\boldsymbol{\Sigma}^{-1} \boldsymbol{d}
$$

Dans un contexte rang faible, le filtre optimal se réduit (Kirsteins et al., 1994; Haimovich, 1997) à :

$$
\boldsymbol{w}_{\text {lropt }}=\boldsymbol{\Pi}_{c}^{\perp} \boldsymbol{d}
$$

La sortie de ce filtre STAP s'exprime comme suit :

$$
\boldsymbol{y}=\left|\boldsymbol{w}_{\text {lropt }}^{H} \boldsymbol{x}\right|^{2}=\left|\boldsymbol{d}^{H} \boldsymbol{\Pi}_{c}^{\perp} \boldsymbol{x}\right|^{2} \propto\left|\boldsymbol{a}(\theta, f)^{H} \boldsymbol{\Pi}_{c}^{\perp} \boldsymbol{x}\right|^{2}
$$

Dans un contexte de maitrise du taux de fausses alarmes, il peut être plus intéressant de construire les différents détecteurs optimaux pour le problème de détection [1] au lieu de calculer uniquement la sortie des filtres [20]. La forme de ces détecteurs dépendra de la connaissance de la puissance du bruit blanc $\lambda$. Même si le modèle du signal est identique à celui présenté dans (Rangaswamy et al., 2004), la procédure pour obtenir les détecteurs est légèrement différente.

Nous appliquons tout d'abord un pre-traitement sur la donnée $\boldsymbol{x}$ en la projetant sur le sous espace orthogonal au sous espace fouillis en utilisant la matrice $\boldsymbol{U}_{0}$ définie dans l'Eq. [7] :

$$
\begin{cases}H_{0}: \boldsymbol{r}=\boldsymbol{U}_{0}^{H} \boldsymbol{x}=\boldsymbol{U}_{0}^{H} \boldsymbol{c}+\boldsymbol{U}_{0}^{H} \boldsymbol{n} & =\boldsymbol{U}_{0}^{H} \boldsymbol{n} \\ H_{1}: \boldsymbol{r}=\boldsymbol{U}_{0}^{H} \boldsymbol{x}=\boldsymbol{U}_{0}^{H} \boldsymbol{d}+\boldsymbol{U}_{0}^{H} \boldsymbol{c}+\boldsymbol{U}_{0}^{H} \boldsymbol{n} & =\boldsymbol{U}_{0}^{H} \boldsymbol{d}+\boldsymbol{U}_{0}^{H} \boldsymbol{n}\end{cases}
$$


Cette étape nous permet de nous débarrasser du fouillis rang faible $c$ quelque soit sa distribution statistique (gaussien ou SIRV). Notons par $\boldsymbol{a}_{0}(\theta, f)=\boldsymbol{U}_{0}^{H} \boldsymbol{a}(\theta, f)$ le vecteur spatio-temporel après projection sur l'orthogonal au sous espace fouillis.

Nous reconnaissons le problème bien connu de détecter un signal complexe $\boldsymbol{d}_{0}=$ $\boldsymbol{U}_{0}^{H} \boldsymbol{d}=\alpha \boldsymbol{a}_{0}(\theta, f)$ corrompu par un bruit additif complexe gaussien centré $\boldsymbol{n}_{0}=$ $\boldsymbol{U}_{0}^{H} \boldsymbol{n} \sim \mathcal{C N}\left(0, \lambda \boldsymbol{I}_{m-r}\right)$ dans $\boldsymbol{r} \in \mathbb{C}^{m-r}:$

$$
\left\{\begin{array}{l}
H_{0}: \boldsymbol{r}=\boldsymbol{n}_{0} \\
H_{1}: \boldsymbol{r}=\boldsymbol{d}_{0}+\boldsymbol{n}_{0}
\end{array}\right.
$$

Pour résoudre le problème de détection [22], nous devons distinguer deux cas : la puissance du bruit blanc $\lambda$ est soit connue soit inconnue.

Dans le premier cas, le test est le matched filter (Robey et al., 1992), qui sera noté dans ce papier le test Low Rank Matched Filter (LRMF) :

$$
\begin{aligned}
\Lambda_{L R M F}(\theta, f) & =\frac{1}{\lambda} \frac{\left|\boldsymbol{a}_{0}(\theta, f)^{H} \boldsymbol{r}\right|^{2}}{\left(\boldsymbol{a}_{0}(\theta, f)^{H} \boldsymbol{a}_{0}(\theta, f)\right)} \underset{H_{0}}{\stackrel{H_{1}}{\gtrless}} \eta \\
& =\frac{1}{\lambda} \frac{\left|\boldsymbol{a}(\theta, f) H^{H} \boldsymbol{\Pi}_{c}^{\perp} \boldsymbol{x}\right|^{2}}{\left(\boldsymbol{a}(\theta, f)^{H} \boldsymbol{\Pi}_{c}^{\perp} \boldsymbol{a}(\theta, f)\right)} \underset{H_{0}}{\stackrel{H_{1}}{\gtrless}} \eta
\end{aligned}
$$

où $\underset{H_{0}}{\stackrel{H_{1}}{\gtrless}} \eta$ signifie que l'hypothèse $H_{1}$ (resp. $H_{0}$ ) est décidée si le test est supérieur (resp. inférieur) au seuil $\eta$.

Dans le second cas, le test est le normalized matched filter (Scharf et al., 1994; Conte et al., 1995; Gini, 1997), qui sera noté dans ce papier le test Low Rank Normalized Matched Filter (LRNMF) :

$$
\begin{aligned}
\Lambda_{L R N M F}(\theta, f) & =\frac{\left|\boldsymbol{a}_{0}(\theta, f)^{H} \boldsymbol{r}\right|^{2}}{\left(\boldsymbol{a}_{0}(\theta, f)^{H} \boldsymbol{a}_{0}(\theta, f)\right)\left(\boldsymbol{r}^{H} \boldsymbol{r}\right)} \stackrel{H_{H_{0}}^{H_{1}}}{\gtrless} \eta \\
& =\frac{\left|\boldsymbol{a}(\theta, f)^{H} \boldsymbol{\Pi}_{c}^{\perp} \boldsymbol{x}\right|^{2}}{\left(\boldsymbol{a}(\theta, f)^{H} \boldsymbol{\Pi}_{c}^{\perp} \boldsymbol{a}(\theta, f)\right)\left(\boldsymbol{x}^{H} \boldsymbol{\Pi}_{c}^{\perp} \boldsymbol{x}\right)} \underset{H_{0}}{\stackrel{H_{1}}{\gtrless}} \eta
\end{aligned}
$$

Le projecteur sur le sous espace fouillis, $\Pi_{c}$, est inconnu et doit être estimateur à partir des données secondaires $\boldsymbol{x}_{k}$ de l'Eq. [1] dans le but de développer des versions adaptatives des tests ci-dessus.

\subsection{Les filtres et les tests de détection optimaux dans un contexte de fouillis rang faible en tenant compte de la propriété de la persymétrie}

Nous présentons tout d'abord le filtre STAP optimal pour le contexte persymétrique :

$$
\boldsymbol{w}_{o p t}^{\prime}=\boldsymbol{\Sigma}^{\prime-1} \boldsymbol{d}^{\prime}
$$


Dans un contexte rang faible, le filtre optimal se réduit (Kirsteins et al., 1994; Haimovich, 1997) à :

$$
\boldsymbol{w}_{\text {lropt }}^{\prime}=\boldsymbol{\Pi}_{c}^{\prime \perp} \boldsymbol{d}^{\prime}
$$

La sortie de ce filtre STAP s'exprime comme suit :

$$
\boldsymbol{y}^{\prime}=\left|\boldsymbol{w}_{\text {lropt }}^{\prime H} \boldsymbol{x}^{\prime}\right|^{2}=\left|\boldsymbol{d}^{\prime H} \boldsymbol{\Pi}_{c}^{\prime \perp} \boldsymbol{x}^{\prime}\right|^{2} \propto\left|\boldsymbol{a}^{\prime H}(\theta, f) \boldsymbol{\Pi}_{c}^{\prime \perp} \boldsymbol{x}^{\prime}\right|^{2}
$$

Comme pour la sous section précédente, nous développons aussi les détecteurs optimaux. Nous appliquons tout d'abord un pre-process sur la donnée $\boldsymbol{x}^{\prime}$ en la projetant sur le sous espace orthogonal au sous espace fouillis en utilisant la matrice $\boldsymbol{U}_{0}^{\prime}$ définie dans l'Eq. [16]:

$$
\begin{cases}H_{0}: \boldsymbol{r}^{\prime}=\boldsymbol{U}_{0}^{\prime H} \boldsymbol{x}^{\prime}=\boldsymbol{U}_{0}^{\prime H} \boldsymbol{c}^{\prime}+\boldsymbol{U}_{0}^{\prime H} \boldsymbol{n}^{\prime} & =\boldsymbol{U}_{0}^{\prime H} \boldsymbol{n}^{\prime} \\ H_{1}: \boldsymbol{r}^{\prime}=\boldsymbol{U}_{0}^{\prime H} \boldsymbol{x}^{\prime}=\boldsymbol{U}_{0}^{\prime H} \boldsymbol{d}^{\prime}+\boldsymbol{U}_{0}^{\prime H} \boldsymbol{c}^{\prime}+\boldsymbol{U}_{0}^{\prime H} \boldsymbol{n}^{\prime}=\boldsymbol{U}_{0}^{\prime H} \boldsymbol{d}^{\prime}+\boldsymbol{U}_{0}^{\prime H} \boldsymbol{n}^{\prime}\end{cases}
$$

Cette étape nous permet de nous débarrasser du fouillis rang faible $\boldsymbol{c}^{\prime}$. Notons par $\boldsymbol{a}_{0}^{\prime}(\theta, f)=\boldsymbol{U}_{0}^{\prime H} \boldsymbol{a}^{\prime}(\theta, f)$ le vecteur spatio-temporel après projection sur l'orthogonal au sous espace fouillis.

Nous reconnaissons le problème bien connu de détecter un signal $\boldsymbol{d}_{0}^{\prime}=\boldsymbol{U}_{0}^{\prime H} \boldsymbol{d}^{\prime}=$ $\alpha \boldsymbol{a}_{0}^{\prime}(\theta, f)$ corrompu par un bruit additif réel gaussien centré $\boldsymbol{n}_{0}^{\prime}=\boldsymbol{U}_{0}^{\prime H} \boldsymbol{n}$ dans $\boldsymbol{r}^{\prime} \in$ $\mathbb{R}^{m-r}:$

$$
\left\{\begin{array}{l}
H_{0}: \boldsymbol{r}^{\prime}=\boldsymbol{n}_{0}^{\prime} \\
H_{1}: \boldsymbol{r}^{\prime}=\boldsymbol{d}_{0}^{\prime}+\boldsymbol{n}_{0}^{\prime}
\end{array}\right.
$$

Pour résoudre le problème de détection [29], nous devons distinguer deux cas : $\lambda$ est soit connue soit inconnue.

Dans le premier cas, le test est le matched filter (Robey et al., 1992), qui sera noté dans ce papier le test Low Rank Matched Filter persymétrique (LRMF') :

$$
\begin{aligned}
\Lambda_{L R M F}^{\prime}(\theta, f) & =\frac{1}{\lambda} \frac{\left|\boldsymbol{a}_{0}^{\prime H}(\theta, f) \boldsymbol{r}^{\prime}\right|^{2}}{\left(\boldsymbol{a}_{0}^{\prime H}(\theta, f) \boldsymbol{a}_{0}^{\prime}(\theta, f)\right)} \underset{H_{0}}{\gtrless} \eta \\
& =\frac{1}{\lambda} \frac{\left|\boldsymbol{a}_{1}^{\prime H}(\theta, f) \boldsymbol{\Pi}_{c}^{\prime \perp} \boldsymbol{x}^{\prime}\right|^{2}}{\left(\boldsymbol{a}^{\prime H}(\theta, f) \boldsymbol{\Pi}_{c}^{\prime \perp} \boldsymbol{a}^{\prime}(\theta, f)\right)} \underset{H_{0}}{\gtrless} \eta \\
& =\frac{1}{\lambda} \frac{\left|\boldsymbol{a}^{H}(\theta, f) \boldsymbol{T}^{H} \boldsymbol{\Pi}_{c}^{\prime \perp} \boldsymbol{T} \boldsymbol{x}\right|^{2}}{\left(\boldsymbol{a}^{H}(\theta, f) \boldsymbol{T}^{H} \boldsymbol{\Pi}_{c}^{\prime \perp} \boldsymbol{T a}(\theta, f)\right)} \underset{H_{0}}{\gtrless} \eta
\end{aligned}
$$

où $\underset{H_{0}}{\stackrel{H_{1}}{\gtrless}} \eta$ signifie que l'hypothèse $H_{1}$ (resp. $H_{0}$ ) est décidée si le test est supérieur (resp. inférieur) au seuil $\eta$. 
Dans le second cas, le test est le normalized matched filter (Scharf et al., 1994; Conte et al., 1995; Gini, 1997), qui sera noté dans ce papier le test Low Rank Normalized Matched Filter persymétrique (LRNMFp) :

$$
\begin{aligned}
\Lambda_{L R N M F}^{\prime}(\theta, f) & =\frac{\left|\boldsymbol{a}_{0}^{\prime H}(\theta, f) \boldsymbol{r}^{\prime}\right|^{2}}{\left(\boldsymbol{a}_{0}^{\prime H}(\theta, f) \boldsymbol{a}_{0}^{\prime}(\theta, f)\right)\left(\boldsymbol{r}^{\prime H} \boldsymbol{r}^{\prime}\right)} \gtrless_{H_{0}}^{H_{1}} \eta \\
& =\frac{\left|\boldsymbol{a}^{\prime H}(\theta, f) \boldsymbol{\Pi}_{c}^{\prime \perp} \boldsymbol{x}^{\prime}\right|^{2}}{\left(\boldsymbol{a}^{\prime H}(\theta, f) \boldsymbol{\Pi}_{c}^{\prime \perp} \boldsymbol{a}^{\prime}(\theta, f)\right)\left(\boldsymbol{x}^{\prime H} \boldsymbol{\Pi}_{c}^{\prime \perp} \boldsymbol{x}^{\prime}\right)} \gtrless_{H_{0}}^{H_{1}} \eta \\
& =\frac{\left|\boldsymbol{a}^{H}(\theta, f) \boldsymbol{T}^{H} \boldsymbol{\Pi}_{c}^{\prime \perp} \boldsymbol{T} \boldsymbol{x}\right|^{2}}{\left(\boldsymbol{a}^{H}(\theta, f) \boldsymbol{T}^{H} \boldsymbol{\Pi}_{c}^{\prime \perp} \boldsymbol{T a}(\theta, f)\right)\left(\boldsymbol{x}^{H} \boldsymbol{T}^{H} \boldsymbol{\Pi}_{c}^{\prime \perp} \boldsymbol{T} \boldsymbol{x}\right)} \gtrless_{H_{0}}^{H_{1}} \eta
\end{aligned}
$$

Le projecteur sur le sous espace fouillis, $\Pi_{c}^{\prime}$, est inconnu et doit être estimateur à partir des données secondaires $\boldsymbol{x}_{k}^{\prime}$ de l'Eq. [13] dans le but de développer des versions adaptatives des tests ci-dessus.

\section{Trois méthodes pour estimer le projecteur orthogonal au sous espace fouillis}

Pour effectuer cette estimation, nous présentons trois méthodes. La première, la plus classique, est basée sur la Sample Covariance Matrix (SCM). La seconde utilise les propriétés éventuelles de symétrie des systèmes STAP et donc la structure persymétrique de la vraie matrice de covariance pour améliorer l'estimation de $\boldsymbol{\Pi}_{c}$. La troisième méthode consiste à utiliser la Normalized Sample Covariance Matrix (NSCM) à la place de la SCM dans le but d'obtenir une meilleure robustesse de l'estimation par rapport à la contamination des données secondaires par des composantes de la cible.

Après une présentation des trois méthodes pour estimer $\boldsymbol{\Pi}_{c}$, nous présentons les résultats des performances des trois filtres STAP sous optimaux résultants de ces trois estimateurs. Ce calcul de performances s'appuie sur une étude du pertes en RSB qui est une grandeur mesurant la perte en rapport signal à bruit si un filtre sous optimal est utilisé à la place du filtre optimal. Une discussion sur la robustesse des estimateurs par rapport à la contamination des données secondaires par des composantes de la cible est aussi donnée en fin de section.

\subsection{Estimateur du projecteur orthogonal au sous espace fouillis}

\subsubsection{Basé sur la SCM}

Il est usuel d'estimer le projecteur $\Pi_{c}^{\perp}$ à partir de la Sample Covariance Matrix $(\mathrm{SCM})$ calculée à partir des $K$ données secondaires $\boldsymbol{x}_{k}$ de l'Eq. [1] :

$$
\hat{\boldsymbol{R}}_{S C M}=\frac{1}{K} \sum_{k=1}^{K} \boldsymbol{x}_{k} \boldsymbol{x}_{k}^{H}
$$


La Décomposition en Valeurs Propres (DVP) de $\hat{\boldsymbol{R}}_{S C M}$ est ensuite appliquée :

$$
\hat{\boldsymbol{R}}_{S C M}=\sum_{i=1}^{r} \hat{\mu}_{i} \hat{\boldsymbol{u}}_{i} \hat{\boldsymbol{u}}_{i}^{H}+\sum_{i=r+1}^{m} \hat{\mu}_{i} \hat{\boldsymbol{u}}_{i} \hat{\boldsymbol{u}}_{i}^{H}
$$

Finalement, nous obtenons l'estimation du projecteur sur le sous espace fouillis (Kirsteins et al., 1994; Haimovich, 1997) :

$$
\begin{aligned}
\hat{\boldsymbol{\Pi}}_{c S C M} & =\sum_{i=1}^{r} \hat{\boldsymbol{u}}_{i} \hat{\boldsymbol{u}}_{i}^{H} \\
\hat{\boldsymbol{\Pi}}_{c S C M}^{\perp} & =\boldsymbol{I}_{m}-\hat{\boldsymbol{\Pi}}_{c S C M}=\sum_{i=r+1}^{m} \hat{\boldsymbol{u}}_{i} \hat{\boldsymbol{u}}_{i}^{H}
\end{aligned}
$$

\subsubsection{Basé sur la SCM et la persymétrie}

La structure persymétrique de $\boldsymbol{\Sigma}$ peut être exploitée pour améliorer la qualité de son estimation comparée à celle utilisant simplement la SCM. Nous pouvons en effet calculer un nouvel estimateur de la vraie matrice de covariance tenant compte de la propriété de persymétrie en utilisant le maximum de vraisemblance. Dans (Pailloux et al., 2008), cet estimateur du maximum de vraisemblance, noté $\hat{\boldsymbol{R}}_{S C M}^{\prime}$, de la vraie matrice de covariance, $\boldsymbol{\Sigma}^{\prime}$, est :

$$
\hat{\boldsymbol{R}}_{S C M}^{\prime}=\mathcal{R} e\left(\hat{\boldsymbol{M}}_{S C M}\right)
$$

où

$$
\hat{\boldsymbol{M}}_{S C M}=\boldsymbol{T} \hat{\boldsymbol{R}}_{S C M} \boldsymbol{T}^{H},
$$

où $\hat{\boldsymbol{R}}_{S C M}$ est la SCM définie dans l'Eq. 32.

La Décomposition en Valeurs Propres (DVP) de $\hat{\boldsymbol{R}}_{S C M}^{\prime}$ est ensuite appliquée :

$$
\hat{\boldsymbol{R}}_{S C M}^{\prime}=\sum_{i=1}^{r} \hat{\mu}_{i}^{\prime} \hat{\boldsymbol{u}}_{i}^{\prime} \hat{\boldsymbol{u}}_{i}^{\prime H}+\sum_{i=r+1}^{m} \hat{\mu}_{i}^{\prime} \hat{\boldsymbol{u}}_{i}^{\prime} \hat{\boldsymbol{u}}_{i}^{\prime H}
$$

Finalement, nous obtenons l'estimation du projecteur sur le sous espace fouillis dans le cas persymétrique (Kirsteins et al., 1994; Haimovich, 1997) :

$$
\begin{aligned}
\hat{\boldsymbol{\Pi}}_{c S C M}^{\prime} & =\sum_{i=1}^{r} \hat{\boldsymbol{u}}_{i}^{\prime} \hat{\boldsymbol{u}}_{i}^{\prime H} \\
\hat{\boldsymbol{\Pi}}_{c S C M}^{\prime \perp} & =\boldsymbol{I}_{m}-\hat{\boldsymbol{\Pi}}_{c S C M}^{\prime}=\sum_{i=r+1}^{m} \hat{\boldsymbol{u}}_{i}^{\prime} \hat{\boldsymbol{u}}_{i}^{\prime H}
\end{aligned}
$$

\subsubsection{Basé sur la NSCM}

Pour le cas d'un bruit modélisé par un processus SIRV sans tenir compte du bruit blanc, d'autres estimations de la matrice de covariance sont utilisées : la NSCM (Gini et al., 1995) et la MPF (Gini et al., 2002; Conte et al., 2002). Puisque le fouillis est supposé être de rang faible, un nombre minimum $2 r$ de données secondaires est nécessaire pour une estimation correcte du projecteur sur le sous espace fouillis, $\Pi_{c}$ (Kirsteins $e t$ al., 1994; Haimovich, 1997). Malheureusement, la procédure itérative pour obtenir la 
MPF dans (Pascal et al., 2008a) doit avoir un nombre d'au moins $N M \gg 2 r$ données secondaires pour des considérations algébriques. Donc, nous proposons dans ce papier de construire le projecteur sur le sous espace fouillis, $\boldsymbol{\Pi}_{c}$, à partir de la NSCM. En effet, même si la NSCM est un estimateur biaisé de la matrice de covariance $\boldsymbol{\Sigma}$, nous avons montré dans (Ginolhac et al., 2011)) que l'estimation du projecteur construit à partir de la NSCM est consistent dans le cas d'un fouillis SIRV plus un bruit blanc gaussien. La NSCM utilisée ensuite pour estimer le projecteur $\Pi_{c}^{\perp}$ s'écrit sous la forme suivante :

$$
\hat{\boldsymbol{R}}_{N S C M}=\frac{m}{K} \sum_{k=1}^{K} \frac{\boldsymbol{x}_{k} \boldsymbol{x}_{k}^{H}}{\boldsymbol{x}_{k}^{H} \boldsymbol{x}_{k}}
$$

La DVP de $\hat{\boldsymbol{R}}_{N S C M}$ s'écrit :

$$
\hat{\boldsymbol{R}}_{N S C M}=\sum_{i=1}^{r} \hat{\mu}_{i} \hat{\boldsymbol{u}}_{i} \hat{\boldsymbol{u}}_{i}^{H}+\sum_{i=r+1}^{m} \hat{\mu}_{i} \hat{\boldsymbol{u}}_{i} \hat{\boldsymbol{u}}_{i}^{H}
$$

Finalement, nous obtenons l'estimation du projecteur sur le sous espace fouillis (Kirsteins et al., 1994; Haimovich, 1997) :

$$
\begin{aligned}
\hat{\boldsymbol{\Pi}}_{c N S C M} & =\sum_{i=1}^{r} \hat{\boldsymbol{u}}_{i} \hat{\boldsymbol{u}}_{i}^{H} \\
\hat{\boldsymbol{\Pi}}_{c N S C M}^{\perp} & =\boldsymbol{I}_{m}-\hat{\boldsymbol{\Pi}}_{c N S C M}=\sum_{i=r+1}^{m} \hat{\boldsymbol{u}}_{i} \hat{\boldsymbol{u}}_{i}^{H}
\end{aligned}
$$

\subsection{Résumé des performances théoriques}

Dans cette sous section, on donne les résultats des performances des filtres STAP sous optimaux construits en utilisant les estimés définis dans les Eq. [34], [38] et [41]. Pour cette étude, nous utilisons le pertes en RSB qui est présenté dans le premier paragraphe. Les trois résultats sont ensuite présentés.

\subsubsection{Définition du pertes en RSB}

Avec les trois estimations du projecteur orthogonal au sous espace fouillis, il est possible de construire trois filtres sous optimaux STAP :

$$
\begin{aligned}
& \hat{\boldsymbol{w}}_{S C M}=\hat{\boldsymbol{\Pi}}_{C S C M}^{\perp} \boldsymbol{d} \\
& \hat{\boldsymbol{w}}_{S C M}^{\prime}=\hat{\boldsymbol{\Pi}}_{c S C M}^{\perp \perp} \boldsymbol{d}^{\prime} \\
& \hat{\boldsymbol{w}}_{N S C M}=\hat{\boldsymbol{\Pi}}_{c N S C M}^{\perp} \boldsymbol{d}
\end{aligned}
$$

Sans tenir compte de la persymétrie, quelque soit le filtre STAP considéré (optimal ou sous optimaux), la sortie du filtre s'exprime comme suit :

$$
\boldsymbol{y}=\boldsymbol{w}^{H} \boldsymbol{x}=\alpha \boldsymbol{w}^{H} \boldsymbol{d}+\boldsymbol{w}^{H} \boldsymbol{c}+\boldsymbol{w}^{H} \boldsymbol{n}
$$

Le Rapport Signal à Bruit à la sortie du filtre $R S B_{\text {out }}$ est :

$$
R S B_{\text {out }}=\frac{|\alpha|^{2}\left|\boldsymbol{w}^{H} \boldsymbol{d}\right|^{2}}{E\left(\boldsymbol{w}^{H}(\boldsymbol{c}+\boldsymbol{n})(\boldsymbol{c}+\boldsymbol{n})^{H} \boldsymbol{w}\right)}=\frac{|\alpha|^{2}\left|\boldsymbol{w}^{H} \boldsymbol{d}\right|^{2}}{\boldsymbol{w}^{H} \boldsymbol{\Sigma} \boldsymbol{w}}
$$


$R S B_{\text {out }}$ est maximum quand $\boldsymbol{w}=\boldsymbol{w}_{\text {opt }}$ de l'Eq. [18] et sa valeur est :

$$
R S B_{\max }=|\alpha|^{2} \boldsymbol{d}^{H} \boldsymbol{\Sigma}^{-1} \boldsymbol{d}
$$

Le pertes en RSB, noté $\rho$, est la perte en performance quand $\boldsymbol{w}=\hat{\boldsymbol{w}}$ de l'Eq. [42]. Son expression est :

$$
\rho=\frac{R S B_{\text {out }}}{R S B_{\max }}=\frac{\left|\hat{\boldsymbol{w}}^{H} \boldsymbol{d}\right|^{2}}{\left(\hat{\boldsymbol{w}}^{H} \boldsymbol{\Sigma} \hat{\boldsymbol{w}}\right)\left(\boldsymbol{d}^{H} \boldsymbol{\Sigma}^{-1} \boldsymbol{d}\right)}
$$

Pour la SCM, nous avons alors :

$$
\rho_{S C M}=\lambda \frac{\left(\boldsymbol{d}^{H} \hat{\boldsymbol{\Pi}}_{c S C M}^{\perp} \boldsymbol{d}\right)^{2}}{\boldsymbol{d}^{H} \hat{\boldsymbol{\Pi}}_{c S C M}^{\perp} \boldsymbol{\Sigma} \hat{\boldsymbol{\Pi}}_{c S C M}^{\perp} \boldsymbol{d}}
$$

Pour la NSCM, nous avons alors :

$$
\rho_{N S C M}=\lambda \frac{\left(\boldsymbol{d}^{H} \hat{\boldsymbol{\Pi}}_{c N S C M}^{\perp} \boldsymbol{d}\right)^{2}}{\boldsymbol{d}^{H} \hat{\boldsymbol{\Pi}}_{c N S C M}^{\perp} \boldsymbol{\Sigma} \hat{\boldsymbol{\Pi}}_{c N S C M}^{\perp} \boldsymbol{d}}
$$

En utilisant les grandeurs persymétriques, on obtient un résultat équivalent pour le pertes en RSB étudiant le filtre STAP rang faible SCM persymétrique :

$$
\rho_{S C M}^{\prime}=\lambda \frac{\left(\boldsymbol{d}^{\prime H} \hat{\boldsymbol{\Pi}}_{c S C M}^{\prime \perp} \boldsymbol{d}^{\prime}\right)^{2}}{\boldsymbol{d}^{\prime H} \hat{\boldsymbol{\Pi}}_{c S C M}^{\prime \perp} \boldsymbol{\Sigma}^{\prime} \hat{\boldsymbol{\Pi}}_{c S C M}^{\perp \perp} \boldsymbol{d}^{\prime}}
$$

Pour étudier théoriquement ces trois pertes en RSB, nous nous basons sur une analyse de perturbation (Krim et al., 1992; Forster et al., 1998). Nous décrivons sommairement les étapes pour obtenir les résultats présentés dans les sous sections suivantes :

- calcul des perturbateurs modélisant l'erreur d'estimation faite sur $\boldsymbol{\Pi}_{c}$ et $\boldsymbol{\Pi}_{c}^{\prime}$,

- remplacer ces formules dans les pertes en RSB [47], 49]] et [48],

- à l'aide de manipulations algébriques et d'approximations de Taylor, simplifier les expressions des pertes en RSB,

- utilisation du théorème de la limite centrale et calcul des moments d'ordre 1 et 2 pour obtenir la distribution statistique des pertes en RSB.

Cette méthode est détaillée précisément dans (Ginolhac, 2011; Ginolhac et al., 2012).

\subsubsection{Performances du filtre STAP rang réduit basé sur la SCM}

En utilisant (Ginolhac, 2011; Ginolhac et al., 2012), nous obtenons la loi du pertes en RSB :

$$
\rho_{S C M}=1-\frac{1}{2 K} \sum_{i=1}^{r} b_{i} \chi_{i}^{2},
$$


où

$$
b_{i}=\left(\frac{E[\tau] \lambda_{i}+\lambda}{E[\tau] \lambda_{i}}\right)^{2}
$$

et $\chi_{i}^{2}$ est une variable aléatoire distribuée selon une loi du Chi2 avec 2 degrés de liberté. Toutes les variables aléatoires $\chi_{i}^{2}$ sont indépendantes.

La valeur finale de la moyenne du pertes en RSB est donc :

$$
E\left[\rho_{S C M}\right]=1-\frac{1}{K} \sum_{i=1}^{r}\left(\frac{E[\tau] \lambda_{i}+\lambda}{E[\tau] \lambda_{i}}\right)^{2}
$$

Dans le cas d'un fort clutter (hypothèse réaliste en STAP), nous avons $E[\tau] \lambda_{i} \gg$ $\lambda$ pour $i=1, \ldots, r$. Alors, l'Eq. [52] peut être réduite à :

$$
E\left[\rho_{S C M}\right] \approx 1-\frac{r}{K}
$$

Nous retrouvons le résultat classique Gaussien de (Kirsteins et al., 1994; Haimovich, 1997). Donc, la texture $\tau$ n'a pas d'influence sur le pertes en RSB $\rho_{S C M}$.

\subsubsection{Performances du filtre STAP rang réduit basé sur la SCM persymétrique}

En utilisant (Ginolhac, 2011; Ginolhac et al., 2012; Pailloux, 2010), nous obtenons la loi du pertes en RSB :

$$
\rho_{S C M}^{\prime}=1-\frac{1}{2 K} \sum_{i=1}^{r} b_{i} \chi_{i}^{1}
$$

où

$$
b_{i}=\left(\frac{E[\tau] \lambda_{i}+\lambda}{E[\tau] \lambda_{i}}\right)^{2}
$$

et $\chi_{i}^{1}$ est une variable aléatoire distribuée selon une loi du Chi2 avec 1 degré de liberté. La différence avec l'Eq. [50] provient du fait que $\hat{\boldsymbol{R}}_{S C M}^{\prime}$ est une matrice réelle.

La valeur finale de la moyenne du pertes en RSB est donc :

$$
E\left[\rho_{S C M}^{\prime}\right]=1-\frac{1}{2 K} \sum_{i=1}^{r}\left(\frac{E[\tau] \lambda_{i}+\lambda}{E[\tau] \lambda_{i}}\right)^{2}
$$

Dans le cas d'un fort clutter (hypothèse réaliste en STAP), nous avons $E[\tau] \lambda_{i} \gg$ $\lambda$ pour $i=1, \ldots, r$. Alors, l'Eq. [52] peut être réduite à :

$$
E\left[\rho_{S C M}^{\prime}\right] \approx 1-\frac{r}{2 K}
$$

En comparant les deux pertes en RSB calculés à partir de la SCM [53] et de la SCM persymétrique [57], la perte de $-3 \mathrm{~dB}$ est atteinte avec $K=r$ dans le cas de la SCM persymétrique au lieu de $K=2 r$ dans le cas classique de la SCM. 


\subsubsection{Performances du filtre STAP rang réduit basé sur la NSCM}

En utilisant (Ginolhac et al., 2012), nous obtenons la loi du pertes en RSB :

$$
\rho_{N S C M}=1-\frac{1}{2 K} \sum_{i=1}^{r} b_{i} \chi_{i}^{2}
$$

où

$$
b_{i}=\frac{E(\tau) \lambda_{i}+\lambda}{\left(\mu_{i}-\mu\right)^{2}} E\left[\frac{\left(\tau_{k} \lambda_{i}+\lambda\right) \chi_{i}^{2} \chi_{r+1}^{2}}{\left(\sum_{j=1}^{r}\left(\tau_{k} \lambda_{j}+\lambda\right) \chi_{j}^{2}+\lambda \chi_{r+2}^{2}(2(m-r))\right)^{2}}\right]
$$

où $\chi_{i}^{2}$ et $\chi_{r+1}^{2}$ sont deux variables du Chi2 à 2 degrés de liberté et $\chi_{r+2}^{2}(2(m-r))$ est une variable du Chi2 à $2(m-r)$ degrés de liberté. Toutes les variables aléatoires sont indépendantes. $\mu_{i}$ et $\mu$ sont les valeurs propres de l'espérance de la NSCM, leur expression est donnée dans (Ginolhac et al., 2011; Ginolhac, 2011). Dans le cas général, il semble difficile d'obtenir une formulation analytique de 1'Eq. [59] et il est donc nécessaire de les évaluer numériquement. Pour une hypothèse d'un fort fouillis gaussien $\left(E[\tau]=1\right.$ et $\lambda_{i} \gg \lambda$ pour $\left.i=1, \ldots, r\right)$, une formulation analytique est donnée dans (Ginolhac et al., 2010).

La valeur finale de la moyenne du pertes en RSB est donc:

$$
E\left[\rho_{N S C M}\right]=1-\frac{1}{K} \sum_{i=1}^{r} b_{i}
$$

Essayons de comprendre le comportement du pertes en RSB $\rho_{N S C M}$ de l'Eq. [60] en fonction de la texture $\tau$ pour un contexte de fort fouillis. Dans (Ginolhac et al., 2011; Ginolhac, 2011), on a montré que $\mu_{i}$ et $\mu$ sont peu dépendants de $\tau$. Le paramètre $E[\tau]$ est constant et n'a donc pas d'influence sur les performances. Mais l'expression dans l'Eq. [59] à l'intérieur de l'espérance dépend clairement de $\frac{1}{\tau}$. Il semble alors logique que les performances de $\rho_{N S C M}$ dépendent des valeurs de la texture $\tau_{k}$.

\subsection{Discussion sur la robustesse des projecteurs orthogonaux au sous espace fouillis construits à partir de la SCM et de la NSCM à la contamination des données secondaires par des composants de la cible}

Dans cette sous section, nous discutons des performances des détecteurs adaptatifs rang faible basés sur la SCM et la NSCM quand les données secondaires sont contaminées par des composants de la cible. Nous ne considérons pas le cas de la persymétrie car elle n'a pas d'influence sur la robustesse.

Dans (Kirsteins et al., 1994; Palka et al., 1998; Ginolhac et al., 2002), les détecteurs adaptatifs rang faible basés sur la SCM ont montré une meilleure robustesse à la 
contamination des données secondaires par des composants de la cible que les détecteurs classiques dans une hypothèse de faible Rapport Signal à fouillis (RSC). Dans ce cas, les composants de la cible sont estimateurs dans l'orthogonal au sous espace fouillis, i.e. ils sont équivalents au bruit blanc. Cette configuration nous permet de garder de bonnes performances en détection avec les détecteurs adaptatifs rang faible basés sur la SCM. Néanmoins, les performances en détection des détecteurs adaptatifs rang faible basés sur la SCM se dégradent fortement quand le RSC augmente.

Pour améliorer cette propriété de robustesse dans le cas d'une forte contamination de la cible dans les données secondaires, le projecteur estimateur à partir de la NSCM est une alternative intéressante au projecteur classique basé sur la SCM. Supposons que $\boldsymbol{x}_{K}$ est fortement contaminé par des composants de la cible ce qui conduit à : $\left\|\boldsymbol{x}_{K}\right\| \gg\left\|\boldsymbol{x}_{1}\right\|, \ldots,\left\|\boldsymbol{x}_{K-1}\right\|$. La SCM

$$
\hat{\boldsymbol{R}}_{S C M}=\frac{1}{K}\left(\boldsymbol{x}_{1} \boldsymbol{x}_{1}^{H}+\ldots+\boldsymbol{x}_{K-1} \boldsymbol{x}_{K-1}^{H}+\boldsymbol{x}_{K} \boldsymbol{x}_{K}^{H}\right)
$$

est évidement très sensible au terme $\boldsymbol{x}_{K} \boldsymbol{x}_{K}^{H}$ étant donné son poids dans la somme. Dans ce cas, les composants de la cible seront estimateurs dans le sous espace fouillis ce qui conduira à la suppression de la cible dans la donnée sous test.

Pour la NSCM

$$
\hat{\boldsymbol{R}}_{N S C M}=\frac{m}{K}\left(\frac{\boldsymbol{x}_{1} \boldsymbol{x}_{1}^{H}}{\left\|\boldsymbol{x}_{1}\right\|^{2}}+\ldots+\frac{\boldsymbol{x}_{K-1} \boldsymbol{x}_{K-1}^{H}}{\left\|\boldsymbol{x}_{K-1}\right\|^{2}}+\frac{\boldsymbol{x}_{K} \boldsymbol{x}_{K}^{H}}{\left\|\boldsymbol{x}_{K}\right\|^{2}}\right)
$$

tous les termes $\frac{\boldsymbol{x}_{k} \boldsymbol{x}_{k}^{H}}{\left\|\boldsymbol{x}_{k}\right\|^{2}}$ ont la même norme de Frobenius (i.e. 1). La NSCM est alors beaucoup moins sensible à la norme importante de la donnée secondaire $\boldsymbol{x}_{K}$. Ceci est équivalent à réduire le RSC dans les données secondaires et donc que les composants de la cible soient estimateurs dans le sous espace bruit blanc. Ainsi le signal dans la donnée sous test ne sera pas supprimé. Donc, les détecteurs rang faible adaptatifs basés sur la NSCM fourniront une meilleure robustesse que les détecteurs rang faible basés sur la SCM.

\section{Détecteurs rang faible adaptatifs}

Les filtres STAP rang faibles adaptatifs (et donc sous optimaux) sont donnés dans l'Eq. [42]. Dans cette section, nous proposons les versions adaptatives basées sur la SCM, la SCM persymétrique et la NSCM des détecteurs rang faible présentés dans les sous sections 2.3 et 2.4

A partir du LRMF de l'Eq. [23] et du LRNMF de l'Eq. [24], nous avons les versions adaptatives basées sur les estimateurs du projecteur de l'Eq. [34] calculées à partir de la SCM (Kirsteins et al., 1994; Haimovich, 1997; Rangaswamy et al., 2004) :

- variance connue :

$$
\hat{\Lambda}_{L R M F-S C M}(\theta, f)=\frac{1}{\lambda} \frac{\left|\boldsymbol{a}(\theta, f)^{H} \hat{\boldsymbol{\Pi}}_{c S C M}^{\perp} \boldsymbol{x}\right|^{2}}{\left(\boldsymbol{a}(\theta, f)^{H} \hat{\boldsymbol{\Pi}}_{c S C M}^{\perp} \boldsymbol{a}(\theta, f)\right)}{\underset{H_{0}}{H_{1}} \lambda}^{H_{1}} \lambda
$$


- variance inconnue :

$$
\hat{\Lambda}_{L R N M F-S C M}(\theta, f)=\frac{\left|\boldsymbol{a}(\theta, f)^{H} \hat{\boldsymbol{\Pi}}_{c S C M}^{\perp} \boldsymbol{x}\right|^{2}}{\left(\boldsymbol{a}(\theta, f)^{H} \hat{\boldsymbol{\Pi}}_{c S C M}^{\perp} \boldsymbol{a}(\theta, f)\right)\left(\boldsymbol{x}^{H} \hat{\boldsymbol{\Pi}}_{c S C M}^{\perp} \boldsymbol{x}\right)}{\underset{H_{0}}{H_{1}} \lambda}_{\gtrless} \lambda
$$

A partir du LRMF persymétrique de l'Eq. [30] et du LRNMF persymétrique de l'Eq. [31], nous avons les versions adaptatives basées sur les estimateurs du projecteur de l'Eq. [38] calculées à partir de la SCM persymétrique (Kirsteins et al., 1994; Haimovich, 1997; Rangaswamy et al., 2004) :

- variance connue :

$$
\hat{\Lambda}_{L R M F-S C M}^{\prime}(\theta, f)=\frac{1}{\lambda} \frac{\left|\boldsymbol{a}(\theta, f)^{H} \boldsymbol{T}^{H} \hat{\boldsymbol{\Pi}}_{c S C M}^{\prime \perp} \boldsymbol{T} \boldsymbol{x}\right|^{2}}{\left(\boldsymbol{a}(\theta, f)^{H} \boldsymbol{T}^{H} \hat{\boldsymbol{\Pi}}_{c S C M}^{\perp} \boldsymbol{a}(\theta, f) \boldsymbol{T}\right)} \underset{H_{0}}{\gtrless} \lambda
$$

- variance inconnue :

$$
\begin{aligned}
& \hat{\Lambda}_{L R N M F-S C M}^{\prime}(\theta, f)= \\
& \frac{\left|\boldsymbol{a}(\theta, f)^{H} \boldsymbol{T}^{H} \hat{\boldsymbol{\Pi}}_{c S C M}^{\prime \perp} \boldsymbol{T} \boldsymbol{x}\right|^{2}}{\left(\boldsymbol{a}(\theta, f)^{H} \boldsymbol{T}^{H} \hat{\boldsymbol{\Pi}}_{c S C M}^{\prime \prime} \boldsymbol{T a}(\theta, f)\right)\left(\boldsymbol{x}^{H} \boldsymbol{T}^{H} \hat{\boldsymbol{\Pi}}_{c S C M}^{\prime \perp} \boldsymbol{T} \boldsymbol{x}\right)} \\
& { }_{H_{0}}^{H_{1}}
\end{aligned}
$$

A partir du LRMF de l'Eq. [23] et du LRNMF de l' Eq. [24], nous avons les versions adaptatives basées sur les estimateurs du projecteur de l'Eq. [41] calculées à partir de la NSCM :

- variance connue :

$$
\hat{\Lambda}_{L R M F-N S C M}(\theta, f)=\frac{1}{\lambda} \frac{\left|\boldsymbol{a}(\theta, f)^{H} \hat{\boldsymbol{\Pi}}_{c N S C M}^{\perp} \boldsymbol{x}\right|^{2}}{\left(\boldsymbol{a}(\theta, f)^{H} \hat{\boldsymbol{\Pi}}_{c N S C M}^{\perp} \boldsymbol{a}(\theta, f)\right)} \underset{H_{0}}{\stackrel{H_{1}}{\gtrless}} \lambda
$$

- variance inconnue :

$$
\hat{\Lambda}_{L R N M F-N S C M}(\theta, f)=\frac{\left|\boldsymbol{a}(\theta, f)^{H} \hat{\boldsymbol{\Pi}}_{c N S C M}^{\perp} \boldsymbol{x}\right|^{2}}{\left(\boldsymbol{a}(\theta, f)^{H} \hat{\boldsymbol{\Pi}}_{c N S C M}^{\perp} \boldsymbol{a}(\theta, f)\right)\left(\boldsymbol{x}^{H} \hat{\boldsymbol{\Pi}}_{c N S C M}^{\perp} \boldsymbol{x}\right)}{\underset{H}{H_{0}}}_{\gtrless}^{H_{1}} \lambda
$$

\section{Résultats}

Cette section s'appuie sur les données DGA/MI présentées dans le papier (Bidon et al., 2011). Dans l'essai 10 considéré, le clutter est obtenu à partir d'une image SAR THR RAMSES et le rapport signal à clutter est égal à $-5 \mathrm{~dB}$. 


\subsection{Configuration}

Dans le scénario considéré, trois cibles sont présentes : $(4 \mathrm{~m} / \mathrm{s}, 0$ deg, cellule 216), ( $4 \mathrm{~m} / \mathrm{s}, 0 \mathrm{deg}$, cellule 256 ) et ( $-4 \mathrm{~m} / \mathrm{s}, 0 \mathrm{deg}$, cellule 296). Nous considérons la cellule 256 sous test. Pour l'intervalle d'entraînement 1 (sans contamination), les données secondaires sont composées des autres cellules valables exceptées les cellules 216 et 296. Pour l'intervalle d'entraînement 2 (avec contamination), les cellules 216 et 296 sont gardées. Dans les deux intervalles d'entraînements, quatre cellules de garde autour de la cellule sous test sont supprimées. Pour conclure, le nombre de données secondaires est $K=408$ dans l'intervalle d'entraînement 1 et $K=410$ dans l'intervalle d'entraînement 2. Pour estimer le rang, nous utilisons la formule de Brennan (Brennan et al., 1992) :

$$
r=N+(M-1) * \beta
$$

Dans notre configuration STAP, la valeur du paramètre $\beta$ est égale à $2 / 3$ et le rang du sous espace fouillis est alors $r=45$. Cette valeur est faible en comparaison à la taille totale de la matrice de covariance du fouillis, $M N=256$.

Comme la puissance du bruit blanc $\lambda$ est inconnue dans cette configuration, les versions normalisées des détecteurs adaptatifs $\hat{\Lambda}_{L R N M F-S C M}$ [64], $\hat{\Lambda}_{L R N M F-S C M}^{\prime}$ 66] et $\hat{\Lambda}_{L R N M F-N S C M}$ 68 sont utilisées.

Pour une référence basique, nous montrons dans la Fig. 1 la sortie du filtre adapté conventionnelle :

$$
\Lambda_{C M F}(\theta, f)=\frac{\left|\boldsymbol{a}(\theta, f)^{H} \boldsymbol{x}\right|^{2}}{m\left(\boldsymbol{x}^{H} \boldsymbol{x}\right)}
$$

Il est calculé pour 64 valeurs de la vitesse de la cible de $-6 \mathrm{~m} / \mathrm{s}$ à $6 \mathrm{~m} / \mathrm{s}$ et 64 valeurs de l'angle d'arrivée de $-3^{\circ}$ à $3^{\circ}$. Ces valeurs sont gardées dans toutes les simulations de cette sous section. Nous notons que la cible à $(4 \mathrm{~m} / \mathrm{s}, 0 \mathrm{deg})$ ne peut pas être détectée à cause du fort fouillis.

Nous utilisons aussi les détecteurs adaptatifs classiques basés sur la SCM et la NSCM. A partir de la SCM de l'Eq. [32], nous avons le détecteur adaptatif suivant (Conte et al., 1995; Scharf et al., 1996) :

$$
\hat{\Lambda}_{N M F-S C M}(\theta, f)=\frac{\left|\boldsymbol{a}(\theta, f)^{H} \hat{\boldsymbol{R}}_{S C M}^{-1} \boldsymbol{x}\right|^{2}}{\left(\boldsymbol{a}(\theta, f)^{H} \hat{\boldsymbol{R}}_{S C M}^{-1} \boldsymbol{a}(\theta, f)\right)\left(\boldsymbol{x}^{H} \hat{\boldsymbol{R}}_{S C M}^{-1} \boldsymbol{x}\right)} \underset{H_{0}}{\stackrel{H_{1}}{\gtrless}} \lambda
$$

A partir de la NSCM de l'Eq. [39], nous avons le détecteur adaptatif suivant (Conte et al., 1996) :

$$
\hat{\Lambda}_{N M F-N S C M}(\theta, f)=\frac{\left|\boldsymbol{a}(\theta, f)^{H} \hat{\boldsymbol{R}}_{N S C M}^{-1} \boldsymbol{x}\right|^{2}}{\left(\boldsymbol{a}(\theta, f)^{H} \hat{\boldsymbol{R}}_{N S C M}^{-1} \boldsymbol{a}(\theta, f)\right)\left(\boldsymbol{x}^{H} \hat{\boldsymbol{R}}_{N S C M}^{-1} \boldsymbol{x}\right)} \underset{H_{0}}{\stackrel{H_{1}}{\gtrless}} \lambda
$$




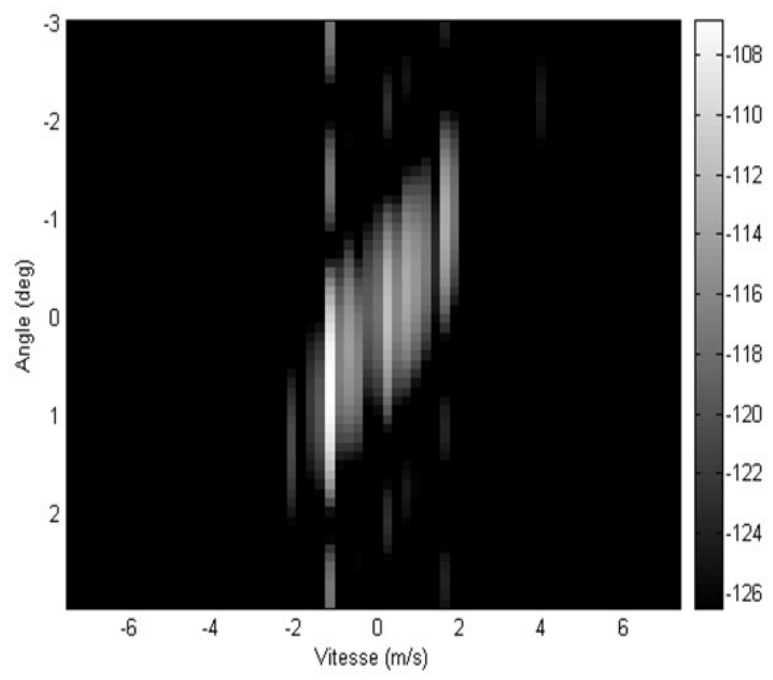

Figure 1. Résultat de détecteur $\Lambda_{C M F}$.

Comme $K<2 N M=512$ et $2 r=90>K$, les performances des détecteurs adaptatifs $\hat{\Lambda}_{N M F-S C M}$ et $\hat{\Lambda}_{N M F-N S C M}$ seront faibles par rapport aux versions rang faible $\hat{\Lambda}_{L R N M F-S C M}, \hat{\Lambda}_{L R N M F-p S C M}$ et $\hat{\Lambda}_{L R N M F-N S C M}$ qui fonctionneront correctement.

\subsection{Apport des Traitements Rang Réduit}

La figure 2 montre les résultats de $\hat{\Lambda}_{N M F-S C M}, \hat{\Lambda}_{N M F-N S C M}$, $\hat{\Lambda}_{L R N M F-S C M}$ et $\hat{\Lambda}_{L R N M F-N S C M}$ pour l'intervalle d'entraînement 1 (sans contamination). Comme le nombre de données secondaires est $K=408<2 N M$, les performances de $\hat{\Lambda}_{N M F-S C M}$ et $\hat{\Lambda}_{N M F-N S C M}$ sont mauvaises comme prévues. Mais la cible peut être détectée à $(4 \mathrm{~m} / \mathrm{s}, 0 \mathrm{deg})$. Les résultats de $\hat{\Lambda}_{L R N M F-S C M}$ et $\hat{\Lambda}_{L R N M F-N S C M}$, sont bien meilleurs car ils nécessitent moins de données secondaires $(2 * r=90)$ pour atteindre de bonnes performances. Comme le fouillis est homogène et qu'il n'y a pas de contamination, leurs deux résultats sont très proches. Cet exemple montre le premier intérêt des approches rang faible qui nécessitent beaucoup moins de données secondaires.

\subsection{Apport de la propriété de persymétrie}

Les figures 3 et 4 montrent les résultats de $\hat{\Lambda}_{L R N M F-S C M}$ et $\hat{\Lambda}_{L R N M F-N S C M}^{\prime}$ pour l'intervalle d'entrainement 1 (sans contamination) avec respectivement 100 

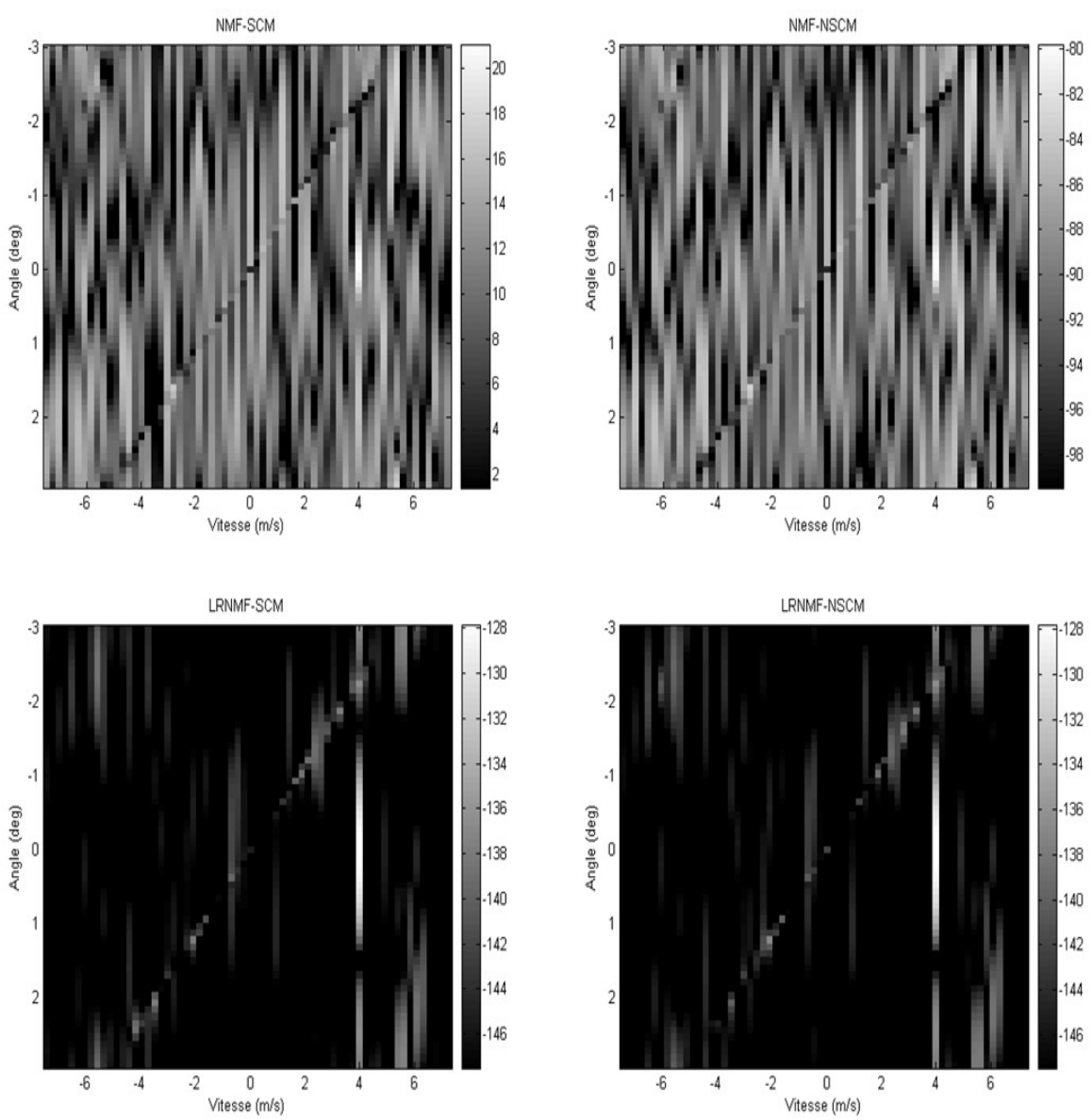

Figure 2. Comparaison des 4 détecteurs $\hat{\Lambda}_{N M F-S C M}$ (en haut à gauche), $\hat{\Lambda}_{N M F-N S C M}$ (en haut à droite), $\hat{\Lambda}_{L R N M F-S C M}$ (en bas à gauche) et $\hat{\Lambda}_{L R-N S C M}$ (en bas à droite) avec l'intervalle d'entraînement 1 (sans contamination).

(presque $2 r$ ) et 50 (presque $r$ ) données secondaires. Comme prévu, nous remarquons que la propriété de persymétrie permet de diminuer le nombre de données secondaires tout en gardant des résultats de détection correct.

\subsection{Apport de la NSCM pour la robustesse}

La figure 5 montre les résultats de $\hat{\Lambda}_{N M F-S C M}, \hat{\Lambda}_{N M F-N S C M}$, $\hat{\Lambda}_{L R N M F-S C M}$ et $\hat{\Lambda}_{L R N M F-N S C M}$ pour l'intervalle d'entraînement 2 (avec contamination). Premièrement, nous pouvons noter que la réponse de la cible n'est 

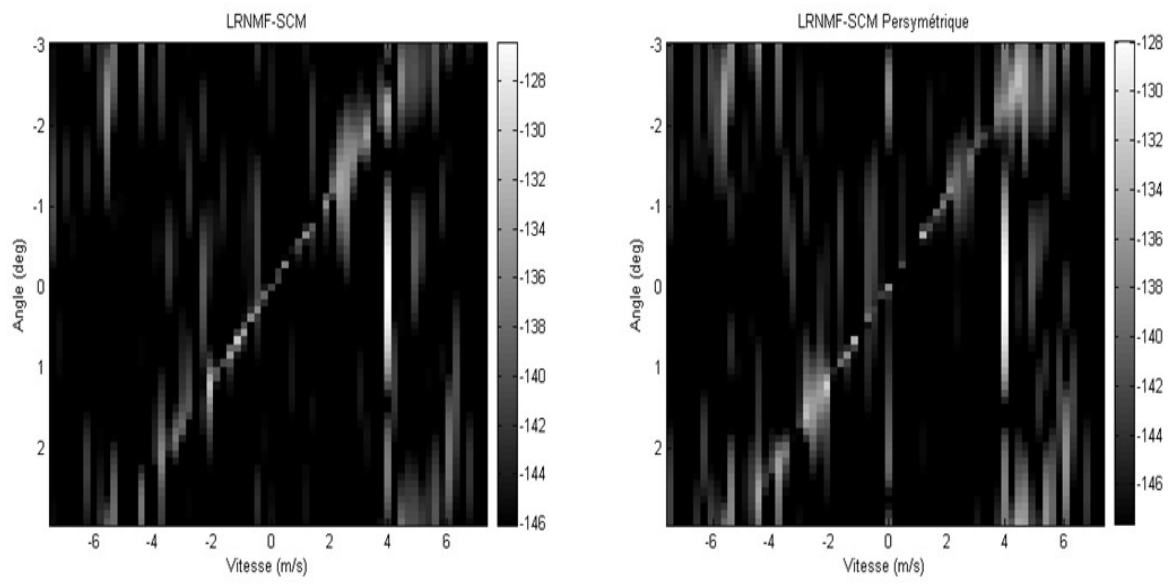

Figure 3. Comparaison des 2 détecteurs $\hat{\Lambda}_{L R N M F-S C M}$ (à gauche) et $\hat{\Lambda}_{L R N M F-S C M}^{\prime}$ (à droite) avec 100 données secondaires de l'intervalle d'entraînement 1 (sans contamination).
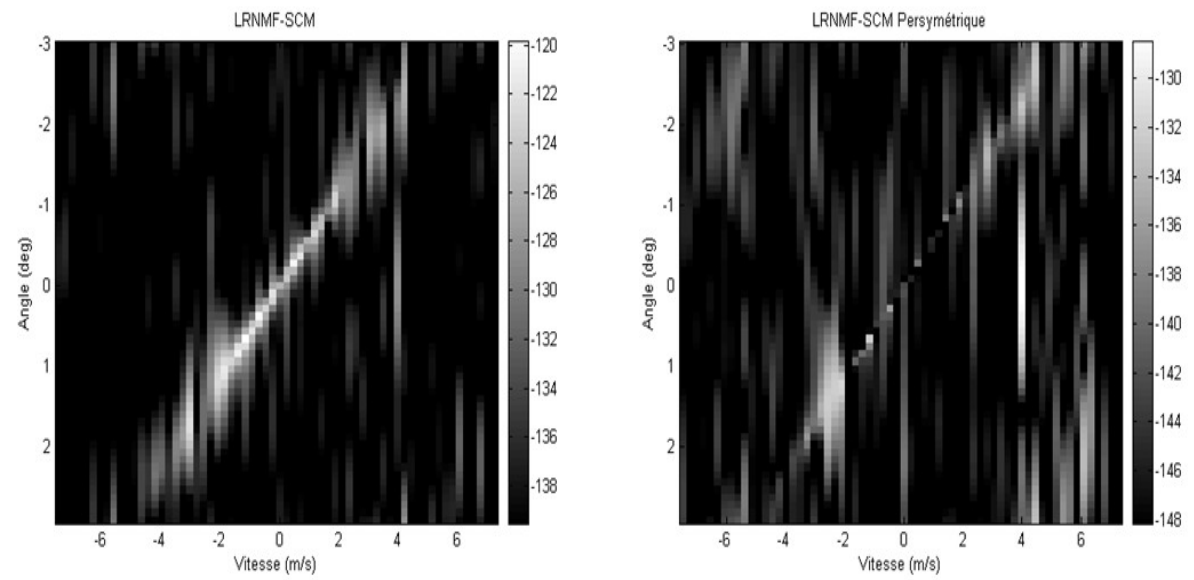

Figure 4. Comparaison des 2 détecteurs $\hat{\Lambda}_{L R N M F-S C M}$ (à gauche) et $\hat{\Lambda}_{L R N M F-S C M}^{\prime}$ (à droite) avec 50 données secondaires de l'intervalle d'entraînement 1 (sans contamination).

plus détectée par $\hat{\Lambda}_{N M F-S C M}$ et $\hat{\Lambda}_{N M F-N S C M}$ à cause de la contamination de la cible dans les données secondaires. Ce résultat est tout à fait logique. Le résultat de $\hat{\Lambda}_{L R N M F-S C M}$ est intéressant : la cible est aussi supprimée et donc nous ne sommes pas dans le cas décrit dans (Kirsteins et al., 1994; Palka et al., 1998; Ginolhac et 
al., 2002) : l'hypothèse de faible RSC n'est pas garantie et donc ne permet pas une bonne robustesse de ce détecteur. Finalement, la performance de notre nouveau détecteur adaptatif, $\hat{\Lambda}_{L R N M F-N S C M}$, est vraiment meilleure : la cible est encore bien détectée et le niveau du fouillis est relativement faible. Cet exemple montre une contribution majeure de la combinaison des méthodes à rang faible et de la NSCM.
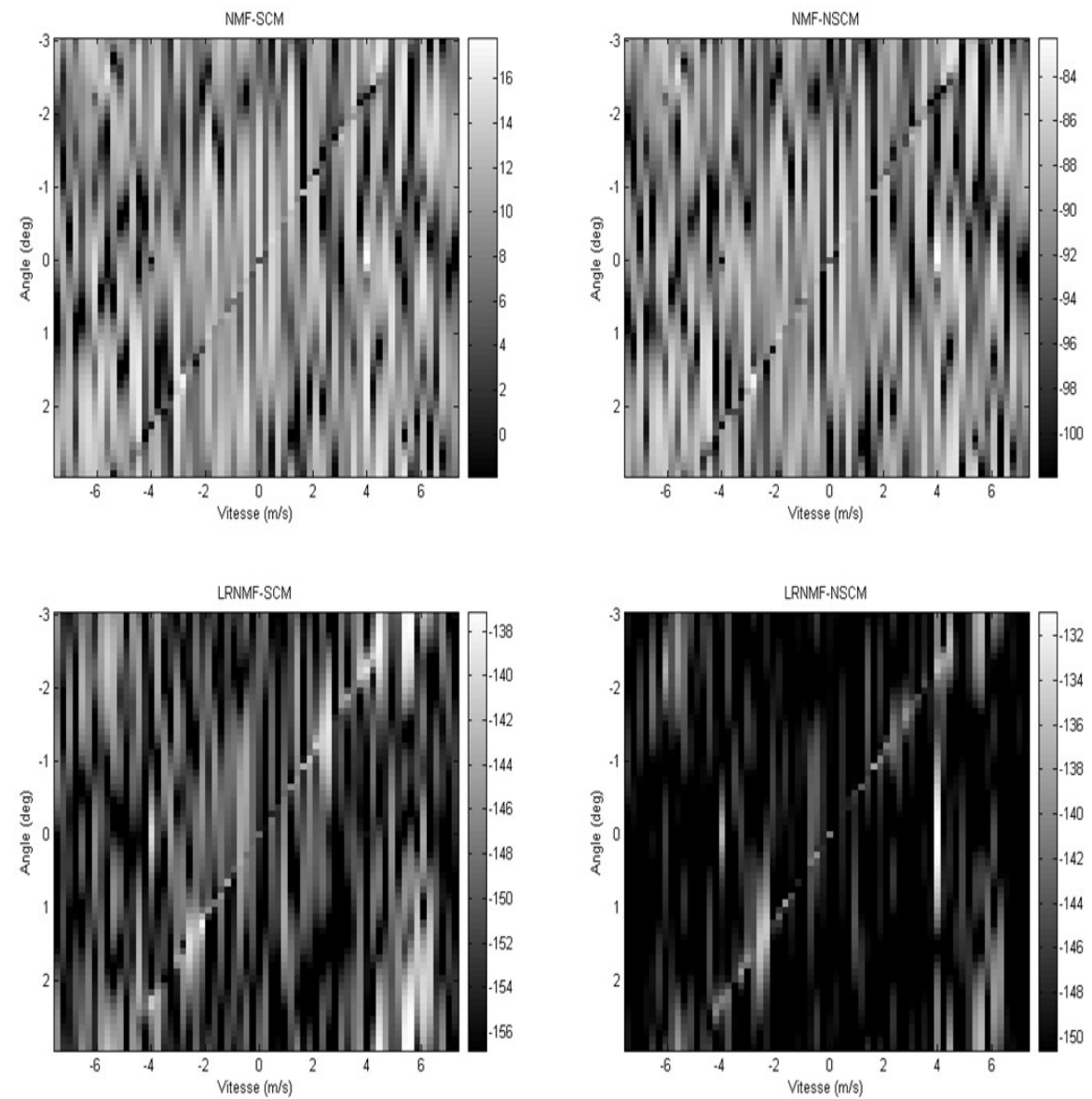

Figure 5. Comparaison des 4 détecteurs $\hat{\Lambda}_{N M F-S C M}$ (en haut à gauche), $\hat{\Lambda}_{N M F-N S C M}\left(\right.$ en haut à droite), $\hat{\Lambda}_{L R N M F-S C M}$ (en bas à gauche) et $\hat{\Lambda}_{L R-N S C M}$ (en bas à droite) avec l'intervalle d'entraînement 2 (avec contamination).

\section{Conclusion}

Dans ce papier, nous avons proposé trois filtres STAP rang faible et les détecteurs adaptatifs correspondants dans le cadre d'un bruit composé d'un fouillis rang faible 
SIRV plus d'un bruit blanc Gaussien. Les projecteurs orthogonaux au sous espace fouillis ont été construits à partir de la SCM (méthode classique), de la SCM persymétrique et de la NSCM. Nous avons présenté les performances de ces trois filtres en terme de pertes en RSB. Tout d'abord, on a retrouvé la propriété de la persymétrie qui permet de réduire le nombre de données secondaires par 2 pour une performance équivalente. On a noté aussi que la texture du SIRV a peu d'influence sur les performances pour les filtres STAP construits à partir de la SCM et de sa version persymétrique. En revanche, elle a une influence sur le filtre construit à partir de la NSCM. Néanmoins, le filtre STAP rang faible construit à partir de la NSCM permet une plus grande robustesse à la contamination des données secondaires par des composants de la cible. Ces différentes propriétés ont été vérifiées sur les données DGA/MI.

Remerciements

Les auteurs souhaitent remercier la DGA/MI pour la fourniture des données STAP utilisées dans cet article.

\section{Bibliographie}

Bidon S., Montecot M., Savy L., « Introduction au SATP. Partie III : Les données du club STAP », Soumis à Traitement du Signal, 2011.

Billingsley J., Ground Clutter Measurements for Surface-Sited Radar, Technical Report nř 780, MIT, February, 1993.

Billingsley J., Farina A., Gini F., Greco M., Verrazzani L., « Statistical analyses of measured radar ground clutter data », IEEE Trans. on Aero. and Elec. Syst., vol. 35, nř 2, p. 579 - 593, 1999.

Brennan L. E., Staudaher F., SubClutter Visibility Demonstration, Technical report, RL-TR-9221, Adaptive Sensors Incorporated, March, 1992.

Cai L., Wang H., « A persymmetric Multiband GLR Algorithm », IEEE Trans. on Aero. and Elec. Syst., vol. 30, nř 3, p. 806-816, July, 1992.

Conte E., Bisceglie M., Galdi C., Ricci G., « A procedure for measuring the coherence length of the sea texture », IEEE Trans. on Instrumentation and Measurement, vol. 46, nř 4, p. 836841, 1997.

Conte E., Lops M., Ricci G., « Asymptotically Optimum Radar Detection in CompoundGaussian Clutter », IEEE Trans. on Aero. and Elec. Syst., vol. 31, nř 2, p. 617 - 625, April, 1995.

Conte E., Lops M., Ricci G., « Adaptive Matched Filter Detection in Spherically Invariant Noise », IEEE Sig. Proc. Letters, August, 1996.

Conte E., Maio A. D., « Exploiting Persymmetry for CFAR Detection in Compound-Gaussian Clutter », IEEE Trans. on Aero. and Elec. Syst., vol. 39, nř 2, p. 719 - 724, April, 2003.

Conte E., Maio A. D., Galdi C., « Statistical analysis of real clutter at different range resolutions », IEEE Trans. on Aero. and Elec. Syst., vol. 40, nř 3, p. 903 - 918, 2004. 
Conte E., Maio A. D., Ricci G., « Recursive Estimation of the Covariance Matrix of a Compound-Gaussian Process and its Application to Adaptive CFAR Detection », IEEE Trans. on Sig. Proc., vol. 50, nř 8, p. 1908 - 1915, August, 2002.

Forster P., Villiers E., Performances asymptotiques des méthodes HR, Les Méthodes à Haute Résolution, S. Marcos, Hermès, chapter 9, p. 245-303, 1998.

Gerlach K., Blunt S., Picciolo M., « Robust Adaptive Matched Filtering using the FRACTA Algorithm », IEEE Trans. on Aero.and Elect. Syst., vol. 40, nř 3, p. 929 - 945, July, 2004.

Gini F., « Sub-Optimum Coherent Radar Detection in a Mixture of K-Distributed and Gaussian Clutter », IEE Proc. Radar, Sonar Navig., vol. 144, nř 1, p. 39 - 48, February, 1997.

Gini F., Greco M., « Covariance Matrix Estimation for CFAR Detection in Correlated Heavy Tailed Clutter », Signal Processing, special section on SP with Heavy Tailed Distributions, vol. 82, nř 12, p. 1847-1859, December, 2002.

Gini F., Greco M., Verrazzani L., « Detection problem in mixed clutter environment as a Gaussian problem by adaptive pre-processing », Electronics Letters, vol. 31, nř 14, p. 1189 1190, July, 1995.

Ginolhac G., Détection / Estimation à l'aide de méthodes algébriques - Application au domaine du RADAR, Rapport HdR, Cachan, France, 2011.

Ginolhac G., Forster P., « Performance Analysis of a Robust Low-Rank STAP Filter in LowRank Gaussian Clutter », Proceedings of ICASSP, Dallas, TX, USA, april, 2010.

Ginolhac G., Forster P., Ovarlez J., Pascal F., « Spatio-temporal adaptive detector in nonhomogeneous and low-rank clutter», Proceedings of ICASSP, Taipei, Taï $\AA_{i}$ wan, april, 2009.

Ginolhac G., Forster P., Pascal F., Ovarlez J., « Derivation of the Bias of the Normalized Sample Covariance Matrix in a Heterogeneous Noise with Application to Low Rank STAP Filter », Submitted in IEEE Trans. on Sig. Proc., 2011.

Ginolhac G., Forster P., Pascal F., Ovarlez J., « Performance and Robustness of two Low-Rank STAP Filters in a Heterogeneous Noise », Submitted in IEEE Trans. on Sig. Proc., 2012.

Ginolhac G., Jourdain G., « "Principal component inverse" algorithm for detection in the presence of reverberation », IEEE Journal of Oceanic Engineering, vol. 27, nř 2, p. 310 - 321, April, 2002.

Greco M., Gini F., Rangaswamy M., « Statistical analysis of measured polarimetric clutter data at different range resolutions », IEE Proceedings on Radar, Sonar and Navigation, vol. 153, nř 6, p. 473 - 481, 2006.

Guerci J., Goldstein J., Reed I., « Optimal and Adaptive Reduced-Rank STAP », IEEE Trans. on Aero.and Elect. Syst., vol. 36, nř 2, p. 647 - 663, April, 2000.

Haimovich A., « The eigencanceler : Adaptive radar by eigenanalysis methods », IEEE Trans. on Aero. and Elec. Syst., vol. 32, nř 2, p. 532 - 542, April, 1996.

Haimovich A., " Asymptotic Distribution of the Conditional Signal-to-Noise Ratio in an Eigenanalysis-Based Adaptive Array », IEEE Trans. on Aero. and Elec. Syst., vol. 33, p. 988 - 997, 1997.

Kirsteins I., Tufts D., Rapidly adaptive nulling of interference, High Resolution Methods in Underwater Acoustics, M. Bouvet and G. Bienvenu, Springer-Verlag, New York, chapter 6, 1991.

Kirsteins I., Tufts D., « Adaptive Detection Using a Low Rank Approximation to a Data Matrix », IEEE Trans. on Aero. and Elec. Syst., vol. 30, p. 55 - 67, 1994. 
Klemm R., Principles of Space-Time Adaptive Processing, Radar, Sonar, Navigation and Avionics, United Kingdom, 2002.

Krim H., Forster P., Proakis J., « Operator approach to performance analysis of root-MUSIC and root-min-norm », IEEE Trans. on Sig. Proc., vol. 40, nř 7, p. 1687 - 1696, July, 1992.

Nohara T., Haykin S., « Canada East Coast Trials and the K-Distribution », IEE Proceeding, Part. F, vol. 138, nř 2, p. 82-88, 1991.

Ovarlez J., Pascal F., Forster P., Giholhac G., Mahot M., « Traitement STAP et modélisation SIRV : Robustesse et Persymétrie », Soumis à Traitement du Signal, 2011.

Pailloux G., Estimation Structurée de la Covariance du Bruit en Détection Adaptative, PhD thesis, Université Paris Ouest La Défense, 2010.

Pailloux G., Forster P., Ovarlez J., Pascal F., « On Persymmetric Covariance Matrices in Adaptive Detection », Proceedings of ICASSP, Las Vegas, Nevada, USA, April, 2008.

Palka T., Tufts D., « Reverberation characterization and suppression by means of Principal Component », Proceedings of OCEANS, Nice, France, September, 1998.

Pascal F., Chitour Y., Ovarlez J., Forster P., Larzabal P., « Existence and Characterization of the Covariance Matrix Maximum Likelihood Estimate in Spherically Invariant Random Processes », IEEE Trans on Sig. Proc., vol. 56, nř 1, p. 34 - 48, January, 2008a.

Pascal F., Forster P., Ovarlez J., Larzabal P., « On a SIRV-CFAR Detector with RADAR Experimentations in Implusive Noise », Proceedings of EUSIPCO, Florence, Italy, september, 2006.

Pascal F., Forster P., Ovarlez J., Larzabal P., « Performance Analysis of Covariance Matrix Estimates in Impulsive Noise », IEEE Trans on Sig. Proc., vol. 56, nř 6, p. 2206 - 2217, June, 2008b.

Peckham C., Haimovich A., T.F.Ãyoub, Goldstein J., Reed I., « Reduced-Rank STAP Performance Analysis », IEEE Trans. on Aero. and Elec. Syst., vol. 36, nř 2, p. 664 - 676, April, 2000.

Rabideau D., Steinhardt A., « Improved Adaptive Clutter Cancellation trough Data-Adaptive Training », IEEE Trans. on Aero. and Elec. Syst., vol. 35, nř 3, p. 879 - 891, July, 1999.

Rangaswamy M., Lin F., Gerlach K., « Robust Adaptive Signal Processing Methods for Heterogeneous Radar Clutter Scenarios », Signal Processing, vol. 84, p. 1653 - 1665, 2004.

Rangaswamy M., Weiner D., Ozturk A., « Non-Gaussian vector identification using spherically invariant random processes », IEEE Trans.-AES, vol. 29, nř 1, p. 111-124, January, 1993.

Reed I., Mallett J., Brennan L., « Rapid Convergence Rate in Adaptive Arrays », IEEE Trans. on Aero. and Elec. Syst., vol. AES-10, nř 6, p. 853 - 863, November, 1974.

Robey F., Fuhrmann D., Kelly E., Nitzberg R., « A CFAR adaptive matched filter detector », IEEE Trans. on Aero. and Elec. Syst., vol. 28, nř 2, p. 208 - 216, 1992.

Scharf L., Friedlander B., « Matched subspace detectors », IEEE Trans. on Sig. Proc., vol. 42, nř 8, p. 2146-2157, august, 1994.

Scharf L., Worther L. M., « Adaptive Matched Subspace Detector and Adaptive Coherence », Proc. of 30th Asilomar Conf. Signals, Syst., Comput., Pacific Grove, CA, USA, November, 1996.

Ward J., Space-Time Adaptive Processing for Airborne Radar, Technical report, Lincoln Lab., MIT, Lexington, Mass., USA, December, 1994. 
Watts S., « Radar detection prediction in sea clutter using the compound K-distribution model », IEE Proceeding, Part. F, vol. 132, nř 7, p. 613-620, December, 1985.

Yao K., «A Representation Theorem and its Applications to Spherically Invariant Random Processes », IEE Trans. on Inf. Th., vol. 19, nř 5, p. 600 - 608, September, 1973. 